\title{
Non-linear multipole interactions and gravitational-wave octupole modes for inspiralling compact binaries to third-and-a-half post-Newtonian order
}

\author{
Guillaume Faye,,$*$ Luc Blanchet,,$\oplus$ and Bala R. Iyer ${ }^{2, \oplus}$ \\ ${ }^{1} \mathcal{G} \mathbb{R} \varepsilon \mathbb{C O}$, Institut d'Astrophysique de Paris - UMR 7095 du CNRS, \\ Université Pierre $\&$ Marie Curie, $98^{\text {bis }}$ boulevard Arago, 75014 Paris, France \\ ${ }^{2}$ Raman Research Institute, Bangalore 560 080, India
}

(Dated: November 16, 2018)

\begin{abstract}
This paper is motivated by the need to improve the post-Newtonian (PN) amplitude accuracy of waveforms for gravitational waves generated by inspiralling compact binaries, both for use in data analysis and in the comparison between post-Newtonian approximations and numerical relativity computations. It presents: (i) the non-linear couplings between multipole moments of general postNewtonian matter sources up to order 3.5PN, including all contributions from tails, tails-of-tails and the non-linear memory effect; and (ii) the source mass-type octupole moment of (non-spinning) compact binaries up to order $3 \mathrm{PN}$, which permits to complete the expressions of the octupole modes $(3,3)$ and $(3,1)$ of the gravitational waveform to order 3.5PN. At this occasion we reconfirm by means of independent calculations our earlier results concerning the source mass-type quadrupole moment to order 3PN. Related discussions on factorized resummed waveforms and the occurence of logarithmic contributions to high order are also included.
\end{abstract}

PACS numbers: 04.25.Nx, 04.30.-w, 97.60.Jd, 97.60.Lf

*Electronic address: faye@iap.fr

$\dagger$ Electronic address: blanchet@iap.fr

${ }^{\ddagger}$ Electronic address: bri@rri.res.in 


\section{INTRODUCTION}

Coalescing compact binaries - two neutron stars or black holes in their late stage of evolution prior the final coalescence - should be the workhorse source driving the network of advanced interferometric gravitational-wave detectors on ground. The post-Newtonian $(\mathrm{PN})$ approximation is the appropriate technique to extract accurate and reliable predictions from general relativity theory for the inspiral phase of these systems. This constitutes the starting point, in data analysis, to construct templates for double neutron-star binaries and a crucial input to validate the early inspiral phase of the numerical relativity waveforms for black-hole binaries.

The non-linear evolution of the orbital phase due to gravitational radiation reaction is the crucial ingredient in constructing these templates. It has been completed for non-spinning compact binaries up to order 3.5PN [1 -7$].{ }^{1}$ The amplitude of the signal, including all signal harmonics besides the dominant one at twice the orbital frequency, has been computed over the years with increasing precision and is now complete to order 3PN [8-12]. Furthermore the dominant quadrupole mode $(2,2)$ is also known to order 3.5PN [13]. Our current program consists in extending this computation and obtaining the full waveform up to order 3.5PN for all the modes $(\ell, m)$ in a spin-weighted spherical-harmonic decomposition. In the present paper we shall, as key milestones for this program:

1. Control all the non-linear couplings between multipole moments up to order 3.5PN for general matter sources; those couplings involve the important contributions of tails, tails-of-tails and the non-linear memory effect, as well as some extra contributions due to our specific definitions for the source multipole moments;

2. Obtain the source mass-type octupole moment of (non-spinning) compact binaries up to order $3 \mathrm{PN}$, which allows us to obtain the expressions of the octupole modes $(3,3)$ and $(3,1)$ of the waveform to order $3.5 \mathrm{PN}$; we shall take this opportunity to recompute, using our new programs, the mass-type quadrupole moment to order $3 \mathrm{PN}$ and confirm the earlier results [5- 7].

The full completion of our program will have to wait for the more difficult computation of the source current-type quadrupole moment to order $3 \mathrm{PN}$, which is left for future work.

The plan of this paper is the following. Sec. II is a recapitulation of the basic definitions we use for source and canonical multipole moments within the multipolar-post-Minkowskian (MPM) formalism. In Sec. III we present (without proof) the expressions of the radiative moments seen at infinity in terms of canonical ones up to order 3.5PN for general matter sources (including the various tail and memory effects), and the explicit links between canonical and source moments. Next, Sec. IV deals with the waveform of non-spinning compact binary sources. Notably, in Subsec. IVB, we compute the mass-type octupole moment for general orbits to 3PN order, reduce it to the center-of-mass frame and then to circular orbits, and, in Subsec. IVC, we obtain the gravitational-wave modes $(3,3)$ and $(3,1)$ up to order 3.5PN for circular orbits. Sec. $\mathrm{V}$ is devoted to the occurence of logarithmic contributions in the MPM waveform to arbitrary high non-linear orders, with an application to factorized resummed waveforms in the effective one body (EOB) approach. The paper ends with three more technical Appendices.

\footnotetext{
${ }^{1}$ As usual the $n \mathrm{PN}$ order refers to the terms of order $1 / c^{2 n}$ in the waveform and energy flux, beyond the Einstein quadrupole formula which is referred to as the Newtonian approximation.
} 


\section{MULTIPOLAR POST-MINKOWSKIAN EXPANSION}

We look for the solution of the Einstein field equations in the vacuum region outside the compact support of a general isolated matter source. With $h^{\alpha \beta} \equiv \sqrt{-g} g^{\alpha \beta}-\eta^{\alpha \beta}$ denoting the "gothic" metric deviation, where $g$ and $g^{\alpha \beta}$ are respectively the determinant and the inverse of the "covariant" metric $g_{\alpha \beta}$ and where $\eta^{\alpha \beta} \equiv \operatorname{diag}(-1,1,1,1)$ stands for the Minkowski metric in Cartesian coordinates, the vacuum field equations relaxed by the harmonic-gauge condition read

$$
\begin{aligned}
\square h^{\alpha \beta} & =\Lambda^{\alpha \beta}\left[h, \partial h, \partial^{2} h\right], \\
\partial_{\beta} h^{\alpha \beta} & =0 .
\end{aligned}
$$

Here $\square \equiv \eta^{\alpha \beta} \partial_{\alpha} \partial_{\beta}$ denotes the flat d'Alembertian operator, whereas the non-linear gravitational source term $\Lambda^{\alpha \beta}$ is an expression of second-order (at least) in the space-time components $h^{\gamma \delta}$, which is quadratic in the first space-time derivatives symbolized by $\partial h$ and linear in the second space-time derivatives $\partial^{2} h$.

The multipolar-post-Minkowskian (MPM) expansion [14] is an algorithmic procedure for generating iteratively the most general solution of the field equations (2.1) in the form of a post-Minkowskian (or non-linearity) expansion whose coefficients are themselves given by a multipole expansion physically valid outside the compact support of the source. The multipole expansion is parametrized by certain multipole moments characterizing the matter source but left, in a first stage, as some unspecified functions of time. However, among these moments, the mass monopole $M$ as well as the mass and current dipoles, $M_{i}$ and $S_{i}$ respectively, are constrained to be constant or to vary linearly with time; they represent the ADM conserved mass, linear momentum and total angular momentum of the source. In this paper we shall work in a mass-centred frame such that $M_{i}=0$. Furthermore, an important assumption of the MPM formalism is the stationarity in the past, namely the fact that the matter source has been stationary in the remote past, before some given date $-\mathcal{T}$. Thus, all multipole moments we shall consider are assumed to be constant when $t \leqslant-\mathcal{T}$.

The starting point of the MPM algorithm is Thorne's [15] linearized vacuum solution parametrized by two types of multipole moments, called the source moments: the mass-type moments $I_{L}(t)$ and the current-type moments $J_{L}(t)$; they are such that $I=M, I_{i}=M_{i}=0$ and $J_{i}=S_{i}$ are constant. Such general linearized solution, referred to as "canonical", reads $^{2}$

\footnotetext{
${ }^{2}$ Our notation is as follows. The retarded time is denoted as $t_{r} \equiv t-r / c$. The $n$-th time derivatives of multipole moments are indicated by superscripts $(n) . L=i_{1} \cdots i_{\ell}$ denotes a multi-index composed of $\ell$ spatial indices (ranging from 1 to 3 ); $a L-1=a i_{1} \cdots i_{\ell-1}$ and so on; $\partial_{L}=\partial_{i_{1}} \cdots \partial_{i_{\ell}}$ is the "product" of $\ell$ partial derivatives $\partial_{i} \equiv \partial / \partial x^{i}$; similarly $x_{L}=x_{i_{1}} \cdots x_{i_{\ell}}$ with $x_{i}$ being the spatial position, and $n_{L}=n_{i_{1}} \cdots n_{i_{\ell}}$ with $n_{i}=x_{i} / r$. Symmetrization over indices is denoted by $T_{(i j)}=\frac{1}{2}\left(T_{i j}+T_{j i}\right)$. The symmetric-trace-free (STF) projection is indicated with a hat, i.e. $\hat{n}_{L} \equiv \operatorname{STF}\left[n_{L}\right]$, or by angular brackets \langle\rangle surrounding the relevant indices, e.g. $\hat{n}_{i j k}=n_{\langle i j k\rangle}=n_{i} n_{j} n_{k}-\frac{1}{5}\left[\delta_{i j} n_{k}+\delta_{j k} n_{i}+\delta_{k i} n_{j}\right]$. Underlined indices mean that they should be excluded from the STF projection, e.g. $T_{\langle\underline{i a j}\rangle}=\frac{1}{2}\left(T_{i a j}+T_{j a i}\right)-\frac{1}{3} \delta_{i j} T_{k a k}$. The multipole moments we use, $\left\{I_{L}, J_{L}, W_{L}, X_{L}, Y_{L}, Z_{L}\right\},\left\{M_{L}, S_{L}\right\}$ and $\left\{U_{L}, V_{L}\right\}$, are all STF, hence e.g. $I_{L}=\hat{I}_{L}=I_{\langle L\rangle}$. In the case of summed-up multi-indices $L$, we do not write the $\ell$ summations from 1 to 3 over the dummy indices. The Levi-Civita antisymmetric symbol is denoted $\varepsilon_{i a b}$ (with $\varepsilon_{123}=1$ ).
} 


$$
\begin{aligned}
& h_{\mathrm{can}(1)}^{00}=-\frac{4}{c^{2}} \sum_{\ell=0}^{+\infty} \frac{(-)^{\ell}}{\ell !} \partial_{L}\left[r^{-1} I_{L}\left(t_{r}\right)\right], \\
& h_{\mathrm{can}(1)}^{0 i}=\frac{4}{c^{3}} \sum_{\ell=1}^{+\infty} \frac{(-)^{\ell}}{\ell !}\left\{\partial_{L-1}\left[r^{-1} I_{i L-1}^{(1)}\left(t_{r}\right)\right]+\frac{\ell}{\ell+1} \varepsilon_{i a b} \partial_{a L-1}\left[r^{-1} J_{b L-1}\left(t_{r}\right)\right]\right\}, \\
& h_{\mathrm{can}(1)}^{i j}=-\frac{4}{c^{4}} \sum_{\ell=2}^{+\infty} \frac{(-)^{\ell}}{\ell !}\left\{\partial_{L-2}\left[r^{-1} I_{i j L-2}^{(2)}\left(t_{r}\right)\right]+\frac{2 \ell}{\ell+1} \partial_{a L-2}\left[r^{-1} \varepsilon_{a b(i} J_{j) b L-2}^{(1)}\left(t_{r}\right)\right]\right\} .
\end{aligned}
$$

It satisfies the relaxed linearized vacuum field equations $\square h_{\text {can (1) }}^{\alpha \beta}=0$ and the harmonic gauge condition $\partial_{\beta} h_{\mathrm{can}(1)}^{\alpha \beta}=0$, formally at any point but $r=0$. However this solution is not the most general one, as we can always perform an arbitrary linearized gauge transformation maintaining the harmonic-gauge condition. Introducing an arbitrary gauge vector $\varphi_{(1)}^{\alpha}$ satisfying $\square \varphi_{(1)}^{\alpha}=0$ (except at $r=0$ ), which will be parametrized by four supplementary types of (unconstrained) multipole moments $W_{L}(t), X_{L}(t), Y_{L}(t)$ and $Z_{L}(t)$ called the gauge moments, we can write

$$
\begin{aligned}
\varphi_{(1)}^{0}= & \frac{4}{c^{3}} \sum_{\ell=0}^{+\infty} \frac{(-)^{\ell}}{\ell !} \partial_{L}\left[r^{-1} W_{L}\left(t_{r}\right)\right], \\
\varphi_{(1)}^{i}= & -\frac{4}{c^{4}} \sum_{\ell=0}^{+\infty} \frac{(-)^{\ell}}{\ell !} \partial_{i L}\left[r^{-1} X_{L}\left(t_{r}\right)\right] \\
& -\frac{4}{c^{4}} \sum_{\ell=1}^{+\infty} \frac{(-)^{\ell}}{\ell !}\left\{\partial_{L-1}\left[r^{-1} Y_{i L-1}\left(t_{r}\right)\right]+\frac{\ell}{\ell+1} \varepsilon_{i a b} \partial_{a L-1}\left[r^{-1} Z_{b L-1}\left(t_{r}\right)\right]\right\} .
\end{aligned}
$$

The linear gauge terms take the form $\partial \varphi_{(1)}^{\alpha \beta} \equiv \partial^{\alpha} \varphi_{(1)}^{\beta}+\partial^{\beta} \varphi_{(1)}^{\alpha}-\eta^{\alpha \beta} \partial_{\gamma} \varphi_{(1)}^{\gamma}$ so that the most general linearized vacuum solution in harmonic coordinates reads

$$
h_{\text {gen (1) }}^{\alpha \beta}=h_{\text {can (1) }}^{\alpha \beta}\left[I_{L}, J_{L}\right]+\partial \varphi_{(1)}^{\alpha \beta}\left[W_{L}, X_{L}, Y_{L}, Z_{L}\right] .
$$

Starting from $h_{\text {gen (1) }}$ the MPM algorithm will generate a full post-Minkowskian solution of the field equations (2.1), i.e. a solution given as a formal non-linear expansion series in powers of Newton's constant $G$, as shown in Eq. (2.10) below. Suppose that one has succeeded in generating all the post-Minkowskian coefficients up to some order $n-1$, say $h_{\text {gen }(2)}, \cdots, h_{\text {gen }(n-1)}$. Then the precise procedure by which the next post-Minkowskian coefficient, i.e. $h_{\text {gen }(n)}$, is generated is as follows [14]. One decomposes this coefficient into two terms,

$$
h_{\operatorname{gen}(n)}^{\alpha \beta}=u_{\operatorname{gen}(n)}^{\alpha \beta}+v_{\operatorname{gen}(n)}^{\alpha \beta} .
$$

The first one is defined as the standard (flat) retarded integral, denoted $\square_{\text {ret }}^{-1}$, of the iterated source term coming from the relaxed Einstein field equation (2.1a). Namely, after obtaining from the previous iterations the $n$-th post-Minkowskian order source term as some $\Lambda_{(n)}=$ $\Lambda_{(n)}\left[h_{\text {gen }(1)}, \cdots, h_{\text {gen }(n-1)}\right]$, we pose

$$
u_{\text {gen }(n)}^{\alpha \beta}=\underset{B=0}{\mathrm{FP}} \square_{\text {ret }}^{-1}\left[\widetilde{r}^{B} \Lambda_{(n)}^{\alpha \beta}\right] .
$$


Crucial to the MPM algorithm is the regularization process based on analytic continuation in a complex parameter $B$ which enters a regulator factor,

$$
\widetilde{r}^{B} \equiv\left(\frac{r}{r_{0}}\right)^{B},
$$

multiplying the source term. Here $r_{0}$ is an arbitrary constant length scale. The regulator (2.7) permits, thanks to analytic continuation, to cure the divergency of the multipole expansion when $r \rightarrow 0$ that follows from the fact that the vacuum solution is physically valid only outside the matter source and is yet to be matched to the actual solution inside it. $^{3}$ Finally, an operation of taking the finite part (FP), i.e. picking up the term with zeroth power of $B$ in the Laurent expansion of the expression when $B \rightarrow 0$, is applied. This fully defines the expression (2.6) as a particular solution of $\square u_{\operatorname{gen}(n)}=\Lambda_{\operatorname{gen}(n)}$ everywhere except at $r=0$.

The second term in Eq. (2.5) ensures that the harmonic gauge condition $\partial_{\beta} h_{\operatorname{gen}(n)}^{\alpha \beta}=0$ is satisfied. It is algorithmically computed from the divergence of the first term, namely $w_{\text {gen }(n)}^{\alpha} \equiv \partial_{\beta} u_{\text {gen }(n)}^{\alpha \beta}$, which is necessarily a retarded solution of the source-free d'Alembertian equation, $\square w_{\text {gen }(n)}^{\alpha}=0$. That solution can thus always be written as

$$
\begin{aligned}
w_{\text {gen }(n)}^{0} & =\sum_{\ell=0}^{+\infty} \partial_{L}\left[r^{-1} N_{L}\left(t_{r}\right)\right], \\
w_{\text {gen }(n)}^{i} & =\sum_{\ell=0}^{+\infty} \partial_{i L}\left[r^{-1} P_{L}\left(t_{r}\right)\right] \\
& +\sum_{\ell=1}^{+\infty}\left\{\partial_{L-1}\left[r^{-1} Q_{i L-1}\left(t_{r}\right)\right]+\varepsilon_{i a b} \partial_{a L-1}\left[r^{-1} R_{b L-1}\left(t_{r}\right)\right]\right\},
\end{aligned}
$$

where the STF multipole moments $\left\{N_{L}, P_{L}, Q_{L}, R_{L}\right\}$ are given by some (very complicated at high post-Minkowskian orders $n$ ) functionals of the initial source and gauge moments $\left\{I_{L}, J_{L}, W_{L}, X_{L}, Y_{L}, Z_{L}\right\}$. We then pose [14, 17]

$$
\begin{aligned}
v_{\text {gen }(n)}^{00}= & -c r^{-1} N^{(-1)}+\partial_{a}\left[r^{-1}\left(-c N_{a}^{(-1)}+c^{2} Q_{a}^{(-2)}-3 P_{a}\right)\right], \\
v_{\text {gen }(n)}^{0 i}= & r^{-1}\left(-c Q_{i}^{(-1)}+3 c^{-1} P_{i}^{(1)}\right)-\varepsilon_{i a b} \partial_{a}\left[r^{-1} c R_{b}^{(-1)}\right]-\sum_{\ell=2}^{+\infty} \partial_{L-1}\left[r^{-1} N_{i L-1}\right], \\
v_{\text {gen }(n)}^{i j}= & -\delta_{i j} r^{-1} P+\sum_{\ell=2}^{+\infty}\left\{2 \delta_{i j} \partial_{L-1}\left[r^{-1} P_{L-1}\right]-6 \partial_{L-2(i}\left[r^{-1} P_{j) L-2}\right]\right. \\
& \left.+\partial_{L-2}\left[r^{-1}\left(c^{-1} N_{i j L-2}^{(1)}+3 c^{-2} P_{i j L-2}^{(2)}-Q_{i j L-2}\right)\right]-2 \partial_{a L-2}\left[r^{-1} \varepsilon_{a b(i} R_{j) b L-2}\right]\right\} .
\end{aligned}
$$

It can readily be checked that $\partial_{\beta} v_{\operatorname{gen}(n)}^{\alpha \beta}=-w_{\operatorname{gen}(n)}^{\alpha}$, hence $\partial_{\beta} h_{\operatorname{gen}(n)}^{\alpha \beta}=0$. Since we also have $\square v_{\operatorname{gen}(n)}^{\alpha \beta}=0$, we see that the $n$-th post-Minkowskian order piece of the gravitational

\footnotetext{
3 The matching to a general isolated post-Newtonian matter source in the external near zone of this source has been elucidated within this formalism in Refs. [16 19].
} 
field (2.5) satisfies the relaxed field equations in harmonic coordinates at order $n$. Note the presence in Eqs. (2.9) of anti-derivatives, denoted e.g. $N^{(-1)}$, which are associated with the secular losses of energy, linear momentum and angular momentum of the source through gravitational radiation.

Finally, we get a full solution of the vacuum Einstein field equations (2.1), parametrized by two sets of source moments $I_{L}, J_{L}$ and four sets of gauge moments $W_{L}, X_{L}, Y_{L}, Z_{L}$, in the form of the post-Minkowskian expansion series

$$
h_{\text {gen }}^{\alpha \beta}=\sum_{n=1}^{+\infty} G^{n} h_{\operatorname{gen}(n)}^{\alpha \beta}\left[I_{L}, J_{L}, W_{L}, X_{L}, Y_{L}, Z_{L}\right] .
$$

It was proved [14] that this represents physically the most general solution of the vacuum field equations outside an isolated matter system. Thanks to the matching, all the multipole moments therein have been given explicit closed-form expressions as integrals over the matter and gravitational fields of a general post-Newtonian source [17, 18].

The explicit MPM construction leading to Eq. (2.10) is quite complicated in practice but now entirely performed on a computer. ${ }^{4}$ It is often convenient to simplify it by considering, instead of the six sets of source and gauge moments, only two, called the canonical masstype and current-type multipole moments, $M_{L}(t)$ and $S_{L}(t)$ respectively. Indeed it has been proved [14, 15] that the most general solution is actually parametrized by two and only two sets of moments - by definition these canonical $M_{L}$ and $S_{L}$ moments. The simplest MPM construction, here referred to as "canonical", is obtained by annulling all the gauge moments in Eq. (2.10) and starting with $M_{L}, S_{L}$ in place of $I_{L}, J_{L}$, i.e.

$$
h_{\mathrm{can}}^{\alpha \beta}=\sum_{n=1}^{+\infty} G^{n} h_{\mathrm{gen}(n)}^{\alpha \beta}\left[M_{L}, S_{L}, 0,0,0,0\right] .
$$

This means that the iteration now begins at linearized order with the solution $h_{\text {can (1) }}\left[M_{L}, S_{L}\right]$. However, even if we proceed with the simpler construction (2.11), we still have to relate the canonical moments $\left\{M_{L}, S_{L}\right\}$ to the source and gauge moments $\left\{I_{L}, J_{L}, W_{L}, X_{L}, Y_{L}, Z_{L}\right\}$, because only the latter are known as explicit integrals over the matter and gravitational fields of the source. To relate these two sets, we impose that the two constructions (2.10) and (2.11) are to be isometric, i.e. to differ by a coordinate transformation. It can be shown - see notably Ref. [12] for an explicit derivation at quadratic order — that this yields unique relations of the type

$$
\begin{aligned}
M_{L} & =I_{L}+\mathcal{M}_{L}[I, J, W, X, Y, Z], \\
S_{L} & =J_{L}+\mathcal{S}_{L}[I, J, W, X, Y, Z],
\end{aligned}
$$

where $\mathcal{M}_{L}$ and $\mathcal{S}_{L}$ denote some non-linear functionals of the source and gauge moments that are at least quadratic and start only at the high order 2.5PN. When the relations (2.12) are satisfied, the two sets of moments $\left\{M_{L}, S_{L}\right\}$ and $\left\{I_{L}, J_{L}, W_{L}, X_{L}, Y_{L}, Z_{L}\right\}$ describe the same physical matter source. We shall give in Sec. IIIB below their most up-to-date explicit forms.

\footnotetext{
4 The MPM algorithm is implemented by using the algebraic computing software Mathematica together with the tensor package $x$ Act [20]. Intermediate results along with the source codes can be provided by the authors on request.
} 


\section{THE RADIATIVE MULTIPOLE MOMENTS}

In the previous section we reviewed the MPM solutions (2.10)-(2.11), which are valid allover the exterior of the source, in particular at future null infinity. However, these solutions exhibit a logarithmic far-zone structure $\sim(\ln r)^{p} / r^{k}$ when expanded as $r \rightarrow+\infty$ with $t_{r} \equiv t-r / c=$ const, where $t$ and $r$ refer to the harmonic coordinates (see also Sec. VAd). This is due to the well-known logarithmic deviation of the null cones with respect to the retarded cones $t-r / c$ in this coordinate grid. It is thus convenient to introduce so-called radiative coordinates $(T, R)$ such that $T_{R} \equiv T-R / c$ is a null coordinate, or becomes asymptotically null in the limit $R \rightarrow+\infty$. We then have (with the angular coordinates being untouched)

$$
T_{R}=t_{r}-\frac{2 G M}{c^{3}} \ln \left(\frac{r}{c b}\right)+\mathcal{O}\left(\frac{1}{r}\right)
$$

where $M$ is the total mass of the source and $b$ is an arbitrary constant time scale, a priori unrelated to the constant $r_{0}$ introduced in the MPM regulator (2.7). In radiative coordinates the structure of the expansion when $R \rightarrow+\infty$ with $T_{R}=$ const is merely $\sim 1 / R^{k}[21]$.

The STF radiative moments $\left\{U_{L}, V_{L}\right\}$ are then defined from the leading $1 / R$ term of the asymptotic waveform by [15]

$$
\begin{aligned}
g_{i j}^{\mathrm{TT}}=\delta_{i j} & +\frac{4 G}{c^{2} R}\left[\sum_{\ell=2}^{+\infty} \frac{1}{c^{\ell} \ell !}\left\{N_{L-2} U_{i j L-2}\left(T_{R}\right)-\frac{2 \ell}{c(\ell+1)} N_{a L-2} \varepsilon_{a b(i} V_{j) b L-2}\left(T_{R}\right)\right\}\right]^{\mathrm{TT}} \\
& +\mathcal{O}\left(\frac{1}{R^{2}}\right)
\end{aligned}
$$

where the superscript TT refers to the usual algebraic transverse-traceless projection. Below we shall present (without the full derivations) the expressions of the radiative multipole moments needed to control the waveform up to order 3.5PN. These results are obtained by implementing the MPM algorithm reviewed in the previous section.

Our goal being to obtain the radiative moments $\left\{U_{L}, V_{L}\right\}$ as functionals of the source and gauge moments $\left\{I_{L}, J_{L}, W_{L}, X_{L}, Y_{L}, Z_{L}\right\}$, it is useful to know beforehand which types of interactions between any moments $A_{J}$ and $B_{K}$ (with $j$ and $k$ indices respectively), among the set of source and gauge moments, are allowed in a given radiative mass moment $U_{L}$ or current moment $V_{L}$ up the $3.5 \mathrm{PN}$ order. To answer that question in the case of quadratic interactions, say $A_{J} \times B_{K}$ where $A_{J}, B_{K} \in\left\{I_{L}, J_{L}, W_{L}, X_{L}, Y_{L}, Z_{L}\right\}$, we have developed some "selection rules" following Refs. [12, 13]. The interactions allowed by those rules at order 3.5PN are given in Table I.

The two panels of Table $\llbracket$ show the maximal number of indices $\ell_{\max }\left[A_{J}, B_{K}\right]$ on the mass moment $U_{L}$ and the current moment $V_{L}$ (respectively), beyond which $U_{L}$ or $V_{L}$ cannot contain products of the two multipole moments $A_{J}$ and $B_{K}$ or their time derivatives (or possibly time anti-derivatives) at the $3.5 \mathrm{PN}$ order in the waveform. Thus, when $\ell>\ell_{\max }\left[A_{J}, B_{K}\right]$ we can safely ignore the multipole interaction $A_{J} \times B_{K}$ in $U_{L}$ or $V_{L}$ since it will be of higher PN order. When $\ell \leqslant \ell_{\max }\left[A_{J}, B_{K}\right]$ we can deduce all the possible relevant interactions $A_{J} \times B_{K}$ by noticing that $j+k=\ell_{\max }\left[A_{J}, B_{K}\right]$ if the product $A_{J} B_{K}$ has the same parity as the radiative moment containing the interaction (i.e. both $A_{J}$ and $B_{K}$ are mass moments or both are current moments in $U_{L}$; one is a mass moment and the other is a current moment 


\begin{tabular}{|l||c|c|c|c|c|c|}
\hline \multicolumn{1}{|c||}{$A_{J}$} & \multicolumn{7}{|c|}{$B_{K}$} \\
\cline { 2 - 7 } & $I_{K}$ & $J_{K}$ & $W_{K}$ & $X_{K}$ & $Y_{K}$ & $Z_{K}$ \\
\hline$I_{J}$ & 6 & 4 & 4 & 2 & 4 & 2 \\
\hline$J_{J}$ & 4 & 4 & 2 & - & 2 & 2 \\
\hline$W_{J}$ & 4 & 2 & 2 & - & 2 & - \\
\hline$X_{J}$ & 2 & - & - & - & - & - \\
\hline$Y_{J}$ & 4 & 2 & 2 & - & 2 & - \\
\hline$Z_{J}$ & 2 & 2 & - & - & - & - \\
\hline
\end{tabular}

\begin{tabular}{|c||c|c|c|c|c|c|}
\hline \multicolumn{1}{|c||}{$A_{J}$} & \multicolumn{7}{|c|}{$B_{K}$} \\
\cline { 2 - 7 } & $I_{K}$ & $J_{K}$ & $W_{K}$ & $X_{K}$ & $Y_{K}$ & $Z_{K}$ \\
\hline$I_{J}$ & 5 & 5 & 3 & - & 3 & 3 \\
\hline$J_{J}$ & 5 & 3 & 3 & - & 3 & - \\
\hline$W_{J}$ & 3 & 3 & - & - & - & - \\
\hline$X_{J}$ & - & - & - & - & - & - \\
\hline$Y_{J}$ & 3 & 3 & - & - & - & - \\
\hline$Z_{J}$ & 3 & - & - & - & - & - \\
\hline
\end{tabular}

TABLE I: Left panel: Values of $\ell_{\max }\left[A_{J}, B_{K}\right]$ for the mass multipole moment $U_{L}$ at the $3.5 \mathrm{PN}$ order for the various possible choices of multipole interactions between $A_{J}$ and $B_{K} \in\{I, J, W, X, Y, Z\}$. Right panel: likewise but for the current multipole moment $V_{L}$. We must have $j+k=\ell_{\max }\left[A_{J}, B_{K}\right]$ if the product $A_{J} B_{K}$ has the same parity as the radiative moment containing the interaction $A_{J} \times B_{K}$, i.e. both $A_{J}$ and $B_{K}$ belong to $\{I, W, X, Y\}$ or both belong to $\{J, Z\}$ in $U_{L}, A_{J}$ belongs to $\{I, W, X, Y\}$ and $B_{K}$ belongs to $\{J, Z\}$ or vice-versa in $V_{L}$; in all other cases $j+k=$ $\ell_{\max }\left[A_{J}, B_{K}\right]+1$. Impossible (because too low) values of $\ell_{\max }\left[A_{J}, B_{K}\right]$ are indicated by dashes.

in $V_{L}$ ), and $j+k=\ell_{\max }\left[A_{J}, B_{K}\right]+1$ in all other cases. ${ }^{5}$ Finally, the case $\ell_{\max }\left[A_{J}, B_{K}\right]<2$ is obviously impossible since radiative moments have at least $\ell=2$. This case is indicated by dashes in the two panels of Table I. We emphasize that the latter rules apply to quadratic interactions, which are the most tricky to control thoroughly. In the present paper we shall also need to include some cubic interactions. These are simpler to look for and will be dealt with separately.

\section{A. Radiative moments in terms of canonical moments}

Like in our previous papers [12, 13], in order to simplify the presentation, we shall first present the radiative moments $\left\{U_{L}, V_{L}\right\}$ in terms of the canonical moments $\left\{M_{L}, S_{L}\right\}$, and only in a second stage shall we give the canonical moments in terms of the set of source and gauge moments $\left\{I_{L}, J_{L}, W_{L}, X_{L}, Y_{L}, Z_{L}\right\}$. Clearly, the selection rules provided in Table【 apply to the full set of quadratic interactions with $A_{J}, B_{K} \in\left\{I_{L}, J_{L}, W_{L}, X_{L}, Y_{L}, Z_{L}\right\}$ as well as to the restricted set with $A_{J}, B_{K} \in\left\{M_{L}, S_{L}\right\}$.

To display the results, it is also convenient to group the terms in the radiative moments $U_{L}$ and $V_{L}$ into different types, namely on the one hand all the instantaneous terms, and on the other hand the hereditary terms [22] which comprise the tails, the tails-of-tails, and the non-linear memory integrals:

$$
\begin{aligned}
& U_{L}=U_{L}^{\text {inst }}+U_{L}^{\text {tail }}+U_{L}^{\text {tail-tail }}+U_{L}^{\text {mem }}+\delta U_{L}, \\
& V_{L}=V_{L}^{\text {inst }}+V_{L}^{\text {tail }}+V_{L}^{\text {tail-tail }}+V_{L}^{\text {mem }}+\delta V_{L} .
\end{aligned}
$$

The terms $\delta U_{L}$ and $\delta V_{L}$ in the above decomposition represent the contributions occuring at $4 \mathrm{PN}$ or higher orders in the waveform (generally with a more complex non-linear structure),

${ }^{5}$ From Eqs. (2.3) we see that the gauge moments $W_{L}, X_{L}$ and $Y_{L}$ have the same parity as the mass moment $I_{L}$, while the gauge moment $Z_{L}$ has the same parity as the current moment $J_{L}$. 
which will be neglected here. Below we shall show the formulas needed to complete the waveform including all its relevant harmonics up to order 3.5PN. Some of those formulas have already been partially published in Refs. [12, 13], but we reproduce them all in order to be self-contained for the convenience of the user.

\section{Instantaneous terms}

These terms, in which by definition all the canonical moments are evaluated at the current (retarded) time $T_{R}=T-R / c$, are the most intricate terms to obtain. For the mass-type moments they are given by:

$$
\begin{aligned}
& U_{i j}^{\mathrm{inst}}=M_{i j}^{(2)} \\
& +\frac{G}{c^{5}}\left[\frac{1}{7} M_{a\langle i}^{(5)} M_{j\rangle a}-\frac{5}{7} M_{a\langle i}^{(4)} M_{j\rangle a}^{(1)}-\frac{2}{7} M_{a\langle i}^{(3)} M_{j\rangle a}^{(2)}+\frac{1}{3} \varepsilon_{a b\langle i} M_{j\rangle a}^{(4)} S_{b}\right] \\
& +\frac{G}{c^{7}}\left[-\frac{1}{432} M_{a b} M_{i j a b}^{(7)}+\frac{1}{432} M_{a b}^{(1)} M_{i j a b}^{(6)}-\frac{5}{756} M_{a b}^{(2)} M_{i j a b}^{(5)}+\frac{19}{648} M_{a b}^{(3)} M_{i j a b}^{(4)}\right. \\
& +\frac{1957}{3024} M_{a b}^{(4)} M_{i j a b}^{(3)}+\frac{1685}{1008} M_{a b}^{(5)} M_{i j a b}^{(2)}+\frac{41}{28} M_{a b}^{(6)} M_{i j a b}^{(1)}+\frac{91}{216} M_{a b}^{(7)} M_{i j a b} \\
& -\frac{5}{252} M_{a b\langle i} M_{j\rangle a b}^{(7)}+\frac{5}{189} M_{a b\langle i}^{(1)} M_{j\rangle a b}^{(6)}+\frac{5}{126} M_{a b\langle i}^{(2)} M_{j\rangle a b}^{(5)}+\frac{5}{2268} M_{a b\langle i}^{(3)} M_{j\rangle a b}^{(4)} \\
& +\frac{5}{42} S_{a} S_{i j a}^{(5)}+\frac{80}{63} S_{a\langle i} S_{j\rangle a}^{(5)}+\frac{16}{63} S_{a\langle i}^{(1)} S_{j\rangle a}^{(4)}-\frac{64}{63} S_{a\langle i}^{(2)} S_{j\rangle a}^{(3)} \\
& +\varepsilon_{a c\langle i}\left(\frac{1}{168} S_{j\rangle b c}^{(6)} M_{a b}+\frac{1}{24} S_{j\rangle b c}^{(5)} M_{a b}^{(1)}+\frac{1}{28} S_{j\rangle b c}^{(4)} M_{a b}^{(2)}+\frac{3}{56} S_{j\rangle b c}^{(2)} M_{a b}^{(4)}\right. \\
& +\frac{187}{168} S_{j\rangle b c}^{(1)} M_{a b}^{(5)}+\frac{65}{84} S_{j\rangle b c} M_{a b}^{(6)}+\frac{1}{189} M_{j\rangle b c}^{(6)} S_{a b}-\frac{1}{189} M_{j\rangle b c}^{(5)} S_{a b}^{(1)} \\
& +\frac{10}{189} M_{j\rangle b c}^{(4)} S_{a b}^{(2)}+\frac{32}{189} M_{j\rangle b c}^{(3)} S_{a b}^{(3)}+\frac{65}{189} M_{j\rangle b c}^{(2)} S_{a b}^{(4)}-\frac{5}{189} M_{j\rangle b c}^{(1)} S_{a b}^{(5)} \\
& \left.\left.-\frac{10}{63} M_{j\rangle b c} S_{a b}^{(6)}-\frac{1}{6} S_{j\rangle b c}^{(3)} M_{a b}^{(3)}\right)\right] \\
& U_{i j k}^{\mathrm{inst}}=M_{i j k}^{(3)} \\
& +\frac{G}{c^{5}}\left[-\frac{4}{3} M_{a\langle i}^{(3)} M_{j k\rangle a}^{(3)}-\frac{9}{4} M_{a\langle i}^{(4)} M_{j k\rangle a}^{(2)}+\frac{1}{4} M_{a\langle i}^{(2)} M_{j k\rangle a}^{(4)}-\frac{3}{4} M_{a\langle i}^{(5)} M_{j k\rangle a}^{(1)}\right. \\
& +\frac{1}{4} M_{a\langle i}^{(1)} M_{j k\rangle a}^{(5)}+\frac{1}{12} M_{a\langle i}^{(6)} M_{j k\rangle a}+\frac{1}{4} M_{a\langle i} M_{j k\rangle a}^{(6)} \\
& +\frac{1}{5} \varepsilon_{a b\langle i}\left(-12 S_{j \underline{a}}^{(2)} M_{k\rangle b}^{(3)}-8 M_{j \underline{a}}^{(2)} S_{k\rangle b}^{(3)}-3 S_{j \underline{a}}^{(1)} M_{k\rangle b}^{(4)}\right. \\
& \left.\left.-27 M_{j \underline{a}}^{(1)} S_{k\rangle b}^{(4)}-S_{j \underline{a}} M_{k\rangle b}^{(5)}-9 M_{j \underline{a}} S_{k\rangle b}^{(5)}-\frac{9}{4} S_{\underline{a}} M_{j k\rangle b}^{(5)}\right)+\frac{12}{5} S_{\langle i} S_{j k\rangle}^{(4)}\right], \\
& U_{i j k l}^{\mathrm{inst}}=M_{i j k l}^{(4)} \\
& +\frac{G}{c^{3}}\left[-\frac{21}{5} M_{\langle i j}^{(5)} M_{k l\rangle}-\frac{63}{5} M_{\langle i j}^{(4)} M_{k l\rangle}^{(1)}-\frac{102}{5} M_{\langle i j}^{(3)} M_{k l\rangle}^{(2)}\right]
\end{aligned}
$$




$$
\begin{aligned}
& +\frac{G}{c^{5}}\left[\frac{7}{55} M_{a\langle i} M_{j k l\rangle a}^{(7)}+\frac{7}{55} M_{a\langle i}^{(1)} M_{j k l\rangle a}^{(6)}+\frac{1}{25} M_{a\langle i}^{(2)} M_{j k l\rangle a}^{(5)}-\frac{28}{11} M_{a\langle i}^{(3)} M_{j k l\rangle a}^{(4)}\right. \\
& -\frac{273}{55} M_{a\langle i}^{(4)} M_{j k l\rangle a}^{(3)}-\frac{203}{55} M_{a\langle i}^{(5)} M_{j k l\rangle a}^{(2)}-\frac{49}{55} M_{a\langle i}^{(6)} M_{j k l\rangle a}^{(1)}+\frac{14}{275} M_{a\langle i}^{(7)} M_{j k l\rangle a} \\
& +\frac{14}{33} M_{a\langle i j} M_{k l\rangle a}^{(7)}+\frac{37}{33} M_{a\langle i j}^{(1)} M_{k l\rangle a}^{(6)}+\frac{9}{11} M_{a\langle i j}^{(2)} M_{k l\rangle a}^{(5)}+\frac{8}{33} M_{a\langle i j}^{(3)} M_{k l\rangle a}^{(4)}+\frac{9}{5} S_{\langle i} S_{j k l\rangle}^{(5)} \\
& +\frac{16}{5} S_{\langle i j} S_{k l\rangle}^{(5)}+\frac{48}{5} S_{\langle i j}^{(1)} S_{k l\rangle}^{(4)}+\frac{32}{5} S_{\langle i j}^{(2)} S_{k l\rangle}^{(3)} \\
& +\varepsilon_{a b\langle i}\left(-\frac{3}{5} M_{j \underline{a}} S_{k l\rangle b}^{(6)}-\frac{63}{25} M_{j \underline{a}}^{(1)} S_{k l\rangle b}^{(5)}+\frac{3}{5} M_{j \underline{a}}^{(2)} S_{k l\rangle b}^{(4)}+\frac{18}{5} M_{j \underline{a}}^{(3)} S_{k l\rangle b}^{(3)}\right. \\
& +\frac{9}{5} M_{j \underline{a}}^{(4)} S_{k l\rangle b}^{(2)}+\frac{3}{5} M_{j \underline{a}}^{(5)} S_{k l\rangle b}^{(1)}+\frac{3}{25} M_{j \underline{a}}^{(6)} S_{k l\rangle b}-\frac{8}{15} S_{j \underline{a}} M_{k l\rangle b}^{(6)}-\frac{24}{25} S_{j \underline{a}}^{(1)} M_{k l\rangle b}^{(5)} \\
& -\frac{8}{5} S_{j \underline{a}}^{(2)} M_{k l\rangle b}^{(4)}+\frac{16}{3} S_{j \underline{a}}^{(3)} M_{k l\rangle b}^{(3)}+\frac{72}{5} S_{j \underline{a}}^{(4)} M_{k l\rangle b}^{(2)}+\frac{56}{5} S_{j \underline{a}}^{(5)} M_{k l\rangle b}^{(1)} \\
& \left.\left.+\frac{232}{75} S_{j \underline{a}}^{(6)} M_{k l\rangle b}+\frac{29}{75} M_{j k l\rangle a}^{(6)} S_{b}\right)\right] \\
& U_{i j k l m}^{\mathrm{inst}}=M_{i j k l m}^{(5)} \\
& +\frac{G}{c^{3}}\left[-\frac{710}{21} M_{\langle i j}^{(3)} M_{k l m\rangle}^{(3)}-\frac{265}{7} M_{\langle i j k}^{(2)} M_{l m\rangle}^{(4)}-\frac{120}{7} M_{\langle i j}^{(2)} M_{k l m\rangle}^{(4)}\right. \\
& \left.-\frac{155}{7} M_{\langle i j k}^{(1)} M_{l m\rangle}^{(5)}-\frac{41}{7} M_{\langle i j}^{(1)} M_{k l m\rangle}^{(5)}-\frac{34}{7} M_{\langle i j k} M_{l m\rangle}^{(6)}-\frac{15}{7} M_{\langle i j} M_{k l m\rangle}^{(6)}\right], \\
& U_{i j k l m n}^{\mathrm{inst}}=M_{i j k l m n}^{(6)} \\
& +\frac{G}{c^{3}}\left[-\frac{45}{28} M_{\langle i j} M_{k l m n\rangle}^{(7)}-\frac{111}{28} M_{\langle i j}^{(1)} M_{k l m n\rangle}^{(6)}-\frac{561}{28} M_{\langle i j}^{(2)} M_{k l m n\rangle}^{(5)}\right. \\
& -\frac{1595}{28} M_{\langle i j}^{(3)} M_{k l m n\rangle}^{(4)}-\frac{2505}{28} M_{\langle i j}^{(4)} M_{k l m n\rangle}^{(3)}-\frac{2115}{28} M_{\langle i j}^{(5)} M_{k l m n\rangle}^{(2)} \\
& -\frac{909}{28} M_{\langle i j}^{(6)} M_{k l m n\rangle}^{(1)}-\frac{159}{28} M_{\langle i j}^{(7)} M_{k l m n\rangle}-\frac{15}{7} M_{\langle i j k} M_{l m n\rangle}^{(7)}-\frac{75}{7} M_{\langle i j k}^{(1)} M_{l m n\rangle}^{(6)} \\
& \left.-\frac{135}{7} M_{\langle i j k}^{(2)} M_{l m n\rangle}^{(5)}-\frac{505}{21} M_{\langle i j k}^{(3)} M_{l m n\rangle}^{(4)}\right] \text {. }
\end{aligned}
$$

In the above expressions, the $1 / c^{5}$ terms in $U_{i j k l}^{\text {inst }}$ and $1 / c^{3}$ terms in $U_{i j k l m n}^{\text {inst }}$ are new with the present paper; the other terms were obtained in Refs. [12, 13]. For the current-type moments we have:

$$
\begin{aligned}
V_{i j}^{\mathrm{inst}}=S_{i j}^{(2)} & \\
+\frac{G}{7 c^{5}}[ & 4 S_{a\langle i}^{(2)} M_{j\rangle a}^{(3)}+8 M_{a\langle i}^{(2)} S_{j\rangle a}^{(3)}+17 S_{a\langle i}^{(1)} M_{j\rangle a}^{(4)}-3 M_{a\langle i}^{(1)} S_{j\rangle a}^{(4)}+9 S_{a\langle i} M_{j\rangle a}^{(5)} \\
& -3 M_{a\langle i} S_{j\rangle a}^{(5)}-\frac{1}{4} S_{a} M_{i j a}^{(5)}-7 \varepsilon_{a b\langle i} S_{\underline{a}} S_{j\rangle b}^{(4)}+\frac{1}{2} \varepsilon_{a c\langle i}\left(3 M_{\underline{a b}}^{(3)} M_{j\rangle b c}^{(3)}+\frac{353}{24} M_{j\rangle b c}^{(2)} M_{a b}^{(4)}\right. \\
& \left.\left.-\frac{5}{12} M_{\underline{a b}}^{(2)} M_{j\rangle b c}^{(4)}+\frac{113}{8} M_{j\rangle b c}^{(1)} M_{a b}^{(5)}-\frac{3}{8} M_{\underline{a b}}^{(1)} M_{j\rangle b c}^{(5)}+\frac{15}{4} M_{j\rangle b c} M_{a b}^{(6)}+\frac{3}{8} M_{\underline{a b}} M_{j\rangle b c}^{(6)}\right)\right],
\end{aligned}
$$

$V_{i j k}^{\mathrm{inst}}=S_{i j k}^{(3)}$ 


$$
\begin{aligned}
& +\frac{G}{c^{3}}\left[\frac{1}{10} \varepsilon_{a b\langle i} M_{j \underline{a}}^{(5)} M_{k\rangle b}-\frac{1}{2} \varepsilon_{a b\langle i} M_{j \underline{a}}^{(4)} M_{k\rangle b}^{(1)}-2 S_{\langle i} M_{j k\rangle}^{(4)}\right] \\
& +\frac{G}{c^{5}}\left[\frac{1}{12} M_{a\langle i} S_{j k\rangle a}^{(6)}+\frac{1}{12} M_{a\langle i}^{(1)} S_{j k\rangle a}^{(5)}+\frac{5}{12} M_{a\langle i}^{(2)} S_{j k\rangle a}^{(4)}+\frac{35}{12} M_{a\langle i}^{(4)} S_{j k\rangle a}^{(2)}+\frac{49}{12} M_{a\langle i}^{(5)} S_{j k\rangle a}^{(1)}\right. \\
& +\frac{19}{12} M_{a\langle i}^{(6)} S_{j k\rangle a}+\frac{2}{27} S_{a\langle i} M_{j k\rangle a}^{(6)}+\frac{10}{27} S_{a\langle i}^{(1)} M_{j k\rangle a}^{(5)}+\frac{2}{27} S_{a\langle i}^{(2)} M_{j k\rangle a}^{(4)}+\frac{8}{9} S_{a\langle i}^{(3)} M_{j k\rangle a}^{(3)} \\
& -\frac{10}{27} S_{a\langle i}^{(4)} M_{j k\rangle a}^{(2)}-\frac{38}{27} S_{a\langle i}^{(5)} M_{j k\rangle a}^{(1)}-\frac{2}{3} S_{a\langle i}^{(6)} M_{j k\rangle a}-\frac{1}{60} S_{a} M_{i j k a}^{(6)} \\
& +\varepsilon_{a b\langle i}\left(-\frac{1}{180} M_{j k\rangle a c}^{(7)} M_{b c}+\frac{11}{900} M_{j k\rangle a c}^{(6)} M_{b c}^{(1)}+\frac{7}{300} M_{j k\rangle a c}^{(5)} M_{b c}^{(2)}\right. \\
& -\frac{37}{270} M_{j k\rangle a c}^{(4)} M_{b c}^{(3)}-\frac{191}{180} M_{j k\rangle a c}^{(3)} M_{b c}^{(4)}-\frac{65}{36} M_{j k\rangle a c}^{(2)} M_{b c}^{(5)}-\frac{367}{300} M_{j k\rangle a c}^{(1)} M_{b c}^{(6)} \\
& -\frac{133}{450} M_{j k>a c} M_{b c}^{(7)}+\frac{1}{27} M_{j \underline{a c}} M_{k\rangle b c}^{(7)}+\frac{5}{162} M_{j \underline{j c}}^{(1)} M_{k\rangle b c}^{(6)}-\frac{5}{162} M_{j \underline{j c}}^{(2)} M_{k\rangle b c}^{(5)} \\
& -\frac{1}{81} M_{j \underline{a c}}^{(3)} M_{k\rangle b c}^{(4)}-\frac{11}{20} S_{j k\rangle b}^{(5)} S_{a}-\frac{88}{45} S_{j \underline{a}} S_{k\rangle b}^{(5)}-\frac{40}{9} S_{j \underline{a}}^{(1)} S_{k\rangle b}^{(4)} \\
& \left.\left.-\frac{32}{9} S_{j \underline{a}}^{(2)} S_{k>b}^{(3)}\right)\right] \text {, }
\end{aligned}
$$

$$
V_{i j k l}^{\mathrm{inst}}=S_{i j k l}^{(4)}
$$$$
\begin{aligned}
+\frac{G}{c^{3}}[ & -\frac{35}{3} S_{\langle i j}^{(2)} M_{k l\rangle}^{(3)}-\frac{25}{3} M_{\langle i j}^{(2)} S_{k l\rangle}^{(3)}-\frac{65}{6} S_{\langle i j}^{(1)} M_{k l\rangle}^{(4)}-\frac{25}{6} M_{\langle i j}^{(1)} S_{k l\rangle}^{(4)}-\frac{19}{6} S_{\langle i j} M_{k l\rangle}^{(5)} \\
& -\frac{11}{6} M_{\langle i j} S_{k l\rangle}^{(5)}-\frac{11}{12} S_{\langle i} M_{j k l\rangle}^{(5)}+\frac{1}{6} \varepsilon_{a b\langle i}\left(-5 M_{j \underline{a}}^{(3)} M_{k l\rangle b}^{(3)}-\frac{11}{2} M_{j \underline{a}}^{(4)} M_{k l\rangle b}^{(2)}-\frac{5}{2} M_{j \underline{a}}^{(2)} M_{k l\rangle b}^{(4)}\right. \\
& \left.\left.-\frac{1}{2} M_{j \underline{a}}^{(5)} M_{k l\rangle b}^{(1)}+\frac{37}{10} M_{j \underline{a}}^{(1)} M_{k l\rangle b}^{(5)}+\frac{3}{10} M_{j \underline{a}}^{(6)} M_{k l\rangle b}+\frac{1}{2} M_{j \underline{a}} M_{k l\rangle b}^{(6)}\right)\right]
\end{aligned}
$$$$
V_{i j k l m}^{\mathrm{inst}}=S_{i j k l m}^{(5)}
$$

$$
\begin{aligned}
+\frac{G}{c^{3}}[ & -\frac{3}{2} M_{\langle i j} S_{k l m\rangle}^{(6)}-\frac{33}{10} M_{\langle i j}^{(1)} S_{k l m\rangle}^{(5)}-12 M_{\langle i j}^{(2)} S_{k l m\rangle}^{(4)}-27 M_{\langle i j}^{(3)} S_{k l m\rangle}^{(3)} \\
& -\frac{69}{2} M_{\langle i j}^{(4)} S_{k l m\rangle}^{(2)}-\frac{39}{2} M_{\langle i j}^{(5)} S_{k l m\rangle}^{(1)}-\frac{21}{5} M_{\langle i j}^{(6)} S_{k l m\rangle}-\frac{4}{3} S_{\langle i j} M_{k l m\rangle}^{(6)} \\
& -\frac{76}{15} S_{\langle i j}^{(1)} M_{k l m\rangle}^{(5)}-\frac{16}{3} S_{\langle i j}^{(2)} M_{k l m\rangle}^{(4)}-8 S_{\langle i j}^{(3)} M_{k l m\rangle}^{(3)}-\frac{28}{3} S_{\langle i j}^{(4)} M_{k l m\rangle}^{(2)} \\
& -\frac{20}{3} S_{\langle i j}^{(5)} M_{k l m\rangle}^{(1)}-\frac{8}{5} S_{\langle i j}^{(6)} M_{k l m\rangle}-\frac{3}{5} S_{\langle i} M_{j k l m\rangle}^{(6)} \\
& +\varepsilon_{a b i i}\left(\frac{1}{14} M_{j \underline{a}} M_{k l m\rangle b}^{(7)}+\frac{1}{2} M_{j \underline{a}}^{(1)} M_{k l m\rangle b}^{(6)}-\frac{3}{5} M_{j \underline{a}}^{(2)} M_{k l m\rangle b}^{(5)}-\frac{4}{3} M_{j \underline{a}}^{(3)} M_{k l m\rangle b}^{(4)}\right. \\
& \quad-\frac{3}{2} M_{j \underline{a}}^{(4)} M_{k l m\rangle b}^{(3)}-\frac{1}{2} M_{j \underline{a}}^{(5)} M_{k l m\rangle b}^{(2)}+\frac{1}{35} M_{j \underline{a}}^{(7)} M_{k l m\rangle b}+\frac{1}{7} M_{j k \underline{a}} M_{l m\rangle b}^{(7)} \\
& \left.\left.+\frac{2}{3} M_{j k \underline{a}}^{(1)} M_{l m\rangle b}^{(6)}+\frac{4}{3} M_{j k \underline{a}}^{(2)} M_{l m\rangle b}^{(5)}+\frac{1}{3} M_{j k \underline{a}}^{(3)} M_{l m\rangle b}^{(4)}\right)\right] .
\end{aligned}
$$

In the expressions for the current moments above, the $1 / c^{5}$ terms in $V_{i j k}^{\text {inst }}$ and $1 / c^{3}$ terms in $V_{i j k l m}^{\text {inst }}$ are new with this paper. For all higher multipole moments it suffices, at this approximation level, to replace $U_{L}^{\text {inst }}$ and $V_{L}^{\text {inst }}$ by the corresponding $M_{L}^{(\ell)}$ and $S_{L}^{(\ell)}$. 


\section{Tail terms}

Next we give the contributions due to tails which correspond to quadratic interactions and arise at (relative) order 1.5PN. They come from the interaction between the mass monopole $M$ and a non-static multipole $M_{L}$ or $S_{L}$ (with $\ell \geqslant 2$ ). For these we dispose of a general formula valid for any $\ell$ [16, 22]. The following contributions have to be added to any mass and current multipole moments:

$$
\begin{aligned}
U_{L}^{\mathrm{tail}}\left(T_{R}\right) & =\frac{2 G M}{c^{3}} \int_{-\infty}^{T_{R}} M_{L}^{(\ell+2)}(\tau)\left[\ln \left(\frac{T_{R}-\tau}{2 b}\right)+\kappa_{\ell}\right] \mathrm{d} \tau, \\
V_{L}^{\mathrm{tail}}\left(T_{R}\right) & =\frac{2 G M}{c^{3}} \int_{-\infty}^{T_{R}} S_{L}^{(\ell+2)}(\tau)\left[\ln \left(\frac{T_{R}-\tau}{2 b}\right)+\pi_{\ell}\right] \mathrm{d} \tau .
\end{aligned}
$$

Here the constant $b$ entering the logarithmic kernel is the constant time scale that has been introduced into the relation (3.1) between the retarded time $T_{R}$ in radiative coordinates and the retarded time $t_{r}$ in harmonic coordinates. The numerical constants $\kappa_{\ell}$ and $\pi_{\ell}$ are given for general $\ell$, in harmonic coordinates, by

$$
\kappa_{\ell}=\frac{2 \ell^{2}+5 \ell+4}{\ell(\ell+1)(\ell+2)}+H_{\ell-2}, \quad \pi_{\ell}=\frac{\ell-1}{\ell(\ell+1)}+H_{\ell-1},
$$

where $H_{k} \equiv \sum_{j=1}^{k} \frac{1}{j}$ denotes the $k$-th harmonic number.

\section{Tail-of-tail terms}

The tails-of-tails arise at relative order $3 \mathrm{PN}$ and are due to the cubic interaction between two monopoles $M$ and one non-static multipole, i.e. $M \times M \times M_{L}$ or $M \times M \times S_{L}$. The tail-of-tail entering the mass quadrupole moment has been already computed in Ref. [2]. Those in the mass octupole and current quadrupole moments, new with this paper, have been obtained by the same method. ${ }^{6}$ The relevant formulas to integrate the elementary cubic source terms are presented in Appendix $\mathrm{A}$, We find

$$
\begin{aligned}
U_{i j}^{\text {tail-tail }}\left(T_{R}\right)= & \frac{G^{2} M^{2}}{c^{6}} \int_{-\infty}^{T_{R}} M_{i j}^{(5)}(\tau)\left[2 \ln ^{2}\left(\frac{T_{R}-\tau}{2 b}\right)+\frac{11}{3} \ln \left(\frac{T_{R}-\tau}{2 b}\right)\right. \\
& \left.-\frac{214}{105} \ln \left(\frac{T_{R}-\tau}{2 \tau_{0}}\right)+\frac{124627}{22050}\right] \mathrm{d} \tau \\
U_{i j k}^{\text {tail-tail }}\left(T_{R}\right)= & \frac{G^{2} M^{2}}{c^{6}} \int_{-\infty}^{T_{R}} M_{i j k}^{(6)}(\tau)\left[2 \ln ^{2}\left(\frac{T_{R}-\tau}{2 b}\right)+\frac{97}{15} \ln \left(\frac{T_{R}-\tau}{2 b}\right)\right. \\
& \left.-\frac{26}{21} \ln \left(\frac{T_{R}-\tau}{2 \tau_{0}}\right)+\frac{13283}{4410}\right] \mathrm{d} \tau \\
V_{i j}^{\text {tail-tail }}\left(T_{R}\right)= & \frac{G^{2} M^{2}}{c^{6}} \int_{-\infty}^{T_{R}} J_{i j}^{(5)}(\tau)\left[2 \ln ^{2}\left(\frac{T_{R}-\tau}{2 b}\right)+\frac{14}{3} \ln \left(\frac{T_{R}-\tau}{2 b}\right)\right.
\end{aligned}
$$

\footnotetext{
${ }^{6}$ Tail-of-tail contributions to the gravitational field have recently been extensively used in a comparison with gravitational self-force results to high post-Newtonian orders [23, 24]. The corresponding sources to the relaxed Einstein equations can be found in Appendix B of Ref. [24].
} 


$$
\left.-\frac{214}{105} \ln \left(\frac{T_{R}-\tau}{2 \tau_{0}}\right)-\frac{26254}{11025}\right] \mathrm{d} \tau
$$

Note the appearance of two constant time scales there: (i) The time scale $b$, which is a pure gauge constant entering the definition of the particular radiative coordinates used in Eq. (3.1). Changing $b$ simply means shifting the origin of time of the radiative coordinate system $\left(T, X^{i}=R N^{i}\right)$ with respect to the harmonic coordinate grid $\left(t, x^{i}=r n^{i}\right)$, which has clearly no physical implication. (ii) The time scale $\tau_{0}=r_{0} / c$, where $r_{0}$ is the regularization constant introduced in the regulator (2.7) of the MPM algorithm; ${ }^{7}$ the constant $\tau_{0}$ cannot be removed by a coordinate transformation, but it must disappear from the radiative moments (and hence from the physical waveform) once the source moments are explicitly related to the parameters of the matter source, e.g. the masses, trajectories and velocities of the particles in a binary system. This has been verified explicitly in the case of the 3PN mass quadrupole moment in Refs. [3, 5]. In Sec. (IV]) below we shall check the cancellation of $\tau_{0}$ in the case of the $3 \mathrm{PN}$ mass octupole moment as well.

Accordingly, there are two types of logarithms in the kernels of Eqs. (3.8): those containing $b$ and those containing $\tau_{0}$. The coefficient of the leading logarithm square (which contains $b$ ) is always 2 . We also know the coefficient of the logarithm containing $\tau_{0}$ in the case of mass-type moments for general $\ell$. Indeed, the coefficient of $\ln \tau_{0}$ in the tails-oftails associated with mass multipole moments, resulting from the long computation of the multipole interactions $M \times M \times M_{L}$, is given by Eq. (A6) in Ref. [25] as

$$
\alpha_{\ell}=2 \frac{15 \ell^{4}+30 \ell^{3}+28 \ell^{2}+13 \ell+24}{\ell(\ell+1)(2 \ell+3)(2 \ell+1)(2 \ell-1)} .
$$

Thus, we have $\alpha_{2}=214 / 105$ and $\alpha_{3}=26 / 21$ in agreement with the coefficients displayed in Eqs. (3.8a) and (3.8b). We shall come back on the significance of this result in Sec. (IV) when we check that the value $\alpha_{3}=26 / 21$ is fully consistent with our 3PN computation of the source mass octupole moment $I_{i j k}$ for compact binaries [see Eqs. (4.12)-(4.13)]. In Sec. $\mathrm{VA}$ we shall investigate the occurence of the dominant powers of logarithms for more general tail interactions of the type $M \times \cdots \times M \times\left(M_{L}\right.$ or $\left.S_{L}\right)$.

\section{Memory terms}

The contributions coming from the non-linear memory effect arise from quadratic interactions between two radiative moments. They have been computed in Refs. [22, 26 30] in the mass quadrupole moment at the lowest order, which is $2.5 \mathrm{PN}$. More recently, the 3.5PN corrections beyond leading order have been obtained for both circular [31] and eccentric orbits [32]. Note that the non-linear memory effect does not enter the current-type multipole moments $V_{L}$, but only the mass-type multipole moments $U_{L}$, hence $V_{L}^{\text {mem }}=0$. Its contribution to the mass radiative moments needed for the 3.5PN waveform read

$$
U_{i j}^{\mathrm{mem}}\left(T_{R}\right)=\frac{G}{c^{5}} \int_{-\infty}^{T_{R}}\left[-\frac{2}{7} M_{a\langle i}^{(3)}(\tau) M_{j\rangle a}^{(3)}(\tau)\right] \mathrm{d} \tau
$$

\footnotetext{
${ }^{7}$ When studying the case of the mass quadrupole tail-of-tail in Ref. [2], the choice $b=\tau_{0}$ was adopted.
} 


$$
\begin{aligned}
+\frac{G}{c^{7}} \int_{-\infty}^{T_{R}}\left[-\frac{5}{756} M_{a b}^{(4)}(\tau) M_{i j a b}^{(4)}(\tau)-\frac{32}{63} S_{a\langle i}^{(3)}(\tau) S_{j\rangle a}^{(3)}(\tau)\right. & \\
& \left.+\varepsilon_{a b\langle i}\left(\frac{5}{42} S_{j\rangle b c}^{(4)}(\tau) M_{a c}^{(3)}(\tau)-\frac{20}{189} M_{j\rangle b c}^{(4)}(\tau) S_{a c}^{(3)}(\tau)\right)\right] \mathrm{d} \tau \\
U_{i j k}^{\mathrm{mem}}\left(T_{R}\right)= & \frac{G}{c^{5}} \int_{-\infty}^{T_{R}}\left[-\frac{1}{3} M_{a\langle i}^{(3)}(\tau) M_{j k\rangle a}^{(4)}(\tau)-\frac{4}{5} \varepsilon_{a b\langle i} M_{j \underline{a}}^{(3)}(\tau) S_{k\rangle b}^{(3)}(\tau)\right] \mathrm{d} \tau \\
U_{i j k l}^{\mathrm{mem}}\left(T_{R}\right)= & \frac{G}{c^{3}} \int_{-\infty}^{T_{R}}\left[\frac{2}{5} M_{\langle i j}^{(3)}(\tau) M_{k l\rangle}^{(3)}(\tau)\right] \mathrm{d} \tau \\
+ & \frac{G}{c^{5}} \int_{-\infty}^{T_{R}}\left[\frac{12}{55} M_{a\langle i}^{(4)}(\tau) M_{j k l\rangle a}^{(4)}(\tau)-\frac{14}{99} M_{a\langle i j}^{(4)}(\tau) M_{k l\rangle a}^{(4)}(\tau)+\frac{32}{45} S_{\langle i j}^{(3)}(\tau) S_{k l\rangle}^{(3)}(\tau)\right. \\
& \left.+\varepsilon_{a b\langle i}\left(-\frac{4}{5} M_{j \underline{a}}^{(3)}(\tau) S_{k l\rangle b}^{(4)}(\tau)+\frac{32}{45} S_{j \underline{a}}^{(3)}(\tau) M_{k l\rangle b}^{(4)}(\tau)\right)\right] \mathrm{d} \tau \\
U_{i j k l m}^{\mathrm{mem}}\left(T_{R}\right)= & \frac{G}{c^{3}} \int_{-\infty}^{T_{R}}\left[\frac{20}{21} M_{\langle i j}^{(3)}(\tau) M_{k l m\rangle}^{(4)}(\tau)\right] \mathrm{d} \tau \\
U_{i j k l m n}^{\mathrm{mem}}\left(T_{R}\right)= & \frac{G}{c^{3}} \int_{-\infty}^{T_{R}}\left[\frac{5}{7} M_{\langle i j k}^{(4)}(\tau) M_{l m n\rangle}^{(4)}(\tau)-\frac{15}{14} M_{\langle i j}^{(4)}(\tau) M_{k l m n\rangle}^{(4)}(\tau)\right] \mathrm{d} \tau
\end{aligned}
$$

In fact, the non-linear memory terms are known for any multipolar order $\ell$ [31]; the above expressions are a particular case of the general formula given for completeness in Appendix C. in which we also present the corresponding modal decomposition of the non-linear memory.

\section{B. Canonical moments in terms of source moments}

Adding up all the previous contributions in Eqs. (3.3) we obtain the radiative mass and current moments $\left\{U_{L}, V_{L}\right\}$ as full functionals of the canonical moments $\left\{M_{L}, S_{L}\right\}$ consistently with our 3.5PN goal. However there still remains to relate with that same precision the canonical moments to the actual sets of source moments $\left\{I_{L}, J_{L}\right\}$ and gauge moments $\left\{W_{L}, X_{L}, Y_{L}, Z_{L}\right\}$ as shown schematically in Eqs. (2.12). Here we present the most complete up-to-date results, with some repetition (for the sake of exhaustiveness) with respect to Refs. [12, 13]:

$$
\begin{aligned}
M_{i j} & =I_{i j}+\frac{4 G}{c^{5}}\left[W^{(2)} I_{i j}-W^{(1)} I_{i j}^{(1)}\right] \\
& +\frac{4 G}{c^{7}}\left[-2 X I_{i j}^{(3)}+\frac{4}{7} I_{a\langle i}^{(3)} W_{j\rangle a}^{(1)}+\frac{6}{7} I_{a\langle i}^{(4)} W_{j\rangle a}-\frac{1}{7} I_{a\langle i} Y_{j\rangle a}^{(3)}-I_{a\langle i}^{(3)} Y_{j\rangle a}+\frac{1}{63} W_{a}^{(3)} I_{i j a}^{(1)}\right. \\
& -\frac{5}{21} W_{a}^{(4)} I_{i j a}+\frac{5}{63} Y_{a}^{(1)} I_{i j a}^{(2)}-\frac{22}{63} Y_{a}^{(2)} I_{i j a}^{(1)}-\frac{25}{21} Y_{a}^{(3)} I_{i j a}+2 W^{(2)} W_{i j}^{(1)} \\
& +2 W^{(3)} W_{i j}+2 W^{(2)} Y_{i j}-\frac{4}{3} W_{\langle i} W_{j\rangle}^{(3)}-4 W_{\langle i} Y_{j\rangle}^{(2)} \\
& \left.+\varepsilon_{a b\langle i}\left(-I_{j\rangle a}^{(3)} Z_{b}+\frac{1}{3} I_{j\rangle a} Z_{b}^{(3)}+\frac{4}{9} J_{j\rangle a} W_{b}^{(3)}+\frac{8}{9} J_{j\rangle a}^{(1)} Y_{b}^{(1)}-\frac{4}{9} J_{j\rangle a} Y_{b}^{(2)}\right)\right]+\mathcal{O}\left(\frac{1}{c^{8}}\right) \\
M_{i j k} & =I_{i j k}+\frac{4 G}{c^{5}}\left[W^{(2)} I_{i j k}-W^{(1)} I_{i j k}^{(1)}+3 I_{\langle i j} Y_{k\rangle}^{(1)}\right]+\mathcal{O}\left(\frac{1}{c^{7}}\right),
\end{aligned}
$$




$$
\begin{aligned}
M_{i j k l} & =I_{i j k l}+\frac{4 G}{c^{5}}\left[-W^{(1)} I_{i j k l}^{(1)}+W^{(2)} I_{i j k l}+4 Y_{\langle i}^{(1)} I_{j k l\rangle}\right]+\mathcal{O}\left(\frac{1}{c^{6}}\right) \\
S_{i j} & =J_{i j}+\frac{2 G}{c^{5}}\left[\varepsilon_{a b\langle i}\left(-I_{j\rangle b}^{(3)} W_{a}-2 I_{j\rangle b} Y_{a}^{(2)}+I_{j\rangle b}^{(1)} Y_{a}^{(1)}\right)+3 J_{\langle i} Y_{j\rangle}^{(1)}-2 J_{i j}^{(1)} W^{(1)}\right] \\
& +\mathcal{O}\left(\frac{1}{c^{7}}\right) \\
S_{i j k} & =J_{i j k}+\frac{4 G}{c^{5}}\left[-W^{(1)} J_{i j k}^{(1)}+\frac{8}{3} Y_{\langle i}^{(1)} J_{j k\rangle}+\varepsilon_{a b\langle i}\left(-\frac{1}{3} I_{j k\rangle a}^{(1)} Y_{b}^{(1)}+I_{j k\rangle a} Y_{b}^{(2)}+I_{j \underline{a}}^{(3)} W_{k\rangle b}\right)\right] \\
& +\mathcal{O}\left(\frac{1}{c^{6}}\right) .
\end{aligned}
$$

The term $1 / c^{7}$ in $M_{i j}$ was already computed in Ref. [13], but the terms $1 / c^{5}$ in $M_{i j k l}$ and $S_{i j k}$ are new with the present paper. Finally, combining the previous formulas (3.11) together with all the results of Sec. IIIA we control the full waveform in terms of the basic source and gauge moments up to order 3.5PN.

\section{GRAVITATIONAL-WAVE OCTUPOLE MODES OF COMPACT BINARIES}

In this section we shall present the mass octupole source moment $I_{i j k}$ of non-spinning compact (point-particle) binaries at 3PN order for general orbits in the center-of-mass frame, as well as the associated octupole gravitational-wave modes. These results are part of our current program to obtain the waveform of compact binaries complete up to order 3.5PN. The mass octupole moment $I_{i j k}$ is not conceptually more difficult than the mass quadrupole moment $I_{i j}$, which has been obtained to order 3PN in Refs. [5 -7] and extended to 3.5PN in Ref. [13]. Therefore, we shall simply present the result of the long calculation, after a short recapitulation of the method, which is based exactly as in Refs. [5 7] on a preliminary Hadamard type self-field regularization, followed by dimensional regularization and renormalization. The more difficult computation of the current quadrupole moment $J_{i j}$ at the $3 \mathrm{PN}$ order will be left for future work.

\section{A. Dimensional regularization of the mass octupole moment}

In the first stage of the calculation we obtain the mass octupole moment by means of the so-called pure-Hadamard-Schwartz (pHS) regularization to deal with the infinite selffield of the point particles. The pHS regularization is a specific, minimal Hadamard-type regularization of integrals, used together with a minimal treatment of contact ambiguities and Schwartz distributional derivatives [33]. It is free of ambiguities but depends on the usual arbitrary UV regularization length scales $s_{A}(A=1,2)$ associated with the Hadamard partie finie regularization of integrals that diverge at the locations of the two point particles [34]. In addition the constant $r_{0}$ introduced into the regulator (2.7) is also involved and plays the role of an IR regularization scale when computing the multipole moments. The result of this initial calculation thus reads

$$
I_{i j k}^{\mathrm{pHS}}=I_{i j k}^{\mathrm{pHS}}\left[\overline{\boldsymbol{y}}_{A}, s_{A}, r_{0}\right]
$$


where we emphasize the dependence on both the UV and IR scales, $s_{A}$ and $r_{0}$ respectively, and the functional dependence on the two trajectories, denoted $\overline{\boldsymbol{y}}_{A}$, with implicit dependence on the associated coordinate velocities $\overline{\boldsymbol{v}}_{A}=\mathrm{d} \overline{\boldsymbol{y}}_{A} / \mathrm{d} t$. The trajectories $\overline{\boldsymbol{y}}_{A}$ will later be understood as being "bare" trajectories to be "dressed" by renormalization [see Eq. (4.3)]. Notice that the initial result (4.1) does not constitute by itself a physical solution to the problem. In order to make it physical within Hadamard's regularization, it must be supplemented by certain "ambiguity terms" [3, 5]. In dimensional regularization [35, 36], which is free of ambiguities and will thus be adopted here, the physical solution is obtained by augmenting the pHS result with some specific "pole part" $\propto 1 /(d-3)$ in the spatial dimension $d$ considered as a complex number [6, 7, 33].

Therefore, in the second stage of the calculation, we add to the pHS result (4.1) the socalled "difference", which is by definition what we precisely have to add in order to obtain the physical result produced by dimensional regularization (DR). The important point is that the latter difference can be computed purely locally, i.e. at the location of the two particles, in the limit where the dimension tends to three, or equivalently $\varepsilon \rightarrow 0$ with $\varepsilon \equiv d-3$, because it is determined only by the singular behaviour of integrals in the neighbourhood of the two singular source points. Moreover, this difference depends on the Hadamard UV regularization scales $s_{A}$ as well as on the DR characteristic parameters, namely $\varepsilon$ and an arbitrary length scale $\ell_{0}$ entering Newton's constant in $d$ dimensions, $G^{(d)}=G \ell_{0}^{\varepsilon}$. To summarize, the mass octupole source moment $I_{i j k}^{\mathrm{DR}}$ in $d=3+\varepsilon$ dimensions is given, in the limit $\varepsilon \rightarrow 0$, by

$$
I_{i j k}^{\mathrm{DR}}\left[\overline{\boldsymbol{y}}_{A}, r_{0}, \varepsilon, \ell_{0}\right]=I_{i j k}^{\mathrm{pHS}}\left[\overline{\boldsymbol{y}}_{A}, s_{A}, r_{0}\right]+\mathcal{D} I_{i j k}\left[\overline{\boldsymbol{y}}_{A}, s_{A}, \varepsilon, \ell_{0}\right]
$$

where the second term is made of a polar part $\propto 1 / \varepsilon$ plus a finite part contribution $\propto \varepsilon^{0}$, i.e. is of the type $\mathcal{D} I_{i j k}=\frac{1}{\varepsilon} A_{i j k}+B_{i j k}+\mathcal{O}(\varepsilon)$, all terms $\mathcal{O}(\varepsilon)$ being systematically neglected. ${ }^{8}$ At this stage we check that the two parameters $s_{A}$ cancel out between the two terms in the right-hand side of (4.2), so that $I_{i j k}^{\mathrm{DR}}$ is free of such arbitrary UV regularization scales.

Finally, in the third stage of the computation, we renormalize the pole part $\propto 1 / \varepsilon$ of the DR result (4.2) by shifting the particle positions that can be thought as the bare trajectories $\overline{\boldsymbol{y}}_{A} \equiv \boldsymbol{y}_{A}^{\text {bare }}$ into some physical positions, corresponding to renormalized trajectories $\boldsymbol{y}_{A} \equiv$ $\boldsymbol{y}_{A}^{\text {renorm }}$ that will entirely absorb the pole. The precise shifts $\boldsymbol{\eta}_{A}$ of the trajectories, such that

$$
\overline{\boldsymbol{y}}_{A}=\boldsymbol{y}_{A}+\boldsymbol{\eta}_{A}\left[\boldsymbol{y}_{A}, r_{A}^{\prime}, \varepsilon, \ell_{0}\right]
$$

will consist of a pole part followed by a finite part, neglecting a remainder $\mathcal{O}(\varepsilon)$. These shifts arise at 3PN order and have been uniquely determined at the same approximation level in Eqs. (1.13) and (6.41)-(6.43) of Ref. [33], or Eq. (6.8) of Ref. [7]. They are precisely those that ensure the complete equivalence between the Hadamard regularized equations of motion - end result of Ref. [37] — and the DR equations of motion obtained in Ref. [33]. Note that the two UV regularization scales $r_{A}^{\prime}$ entering Eqs. (4.3) are a priori different from the parameters $s_{A}$; they have been chosen instead to match exactly their counterparts entering the Hadamard regularized 3PN equations of motion [37], in which they play the role of UV regularization scales in the context of Hadamard's regularization. Finally our physical, renormalized ("dressed") result, which is numerically equal to the original, bare

\footnotetext{
${ }^{8}$ One can show that there is only a simple pole at order 3PN.
} 
result, modulo $\mathcal{O}(\varepsilon)$ corrections, i.e.

$$
I_{i j k}\left[\boldsymbol{y}_{A}, r_{A}^{\prime}, r_{0}\right] \equiv I_{i j k}^{\mathrm{DR}}\left[\overline{\boldsymbol{y}}_{A}, r_{0}, \varepsilon, \ell_{0}\right]+\mathcal{O}(\varepsilon),
$$

is finite in the limit where $\varepsilon \rightarrow 0$ while keeping the dressed trajectories $\boldsymbol{y}_{A}$ constant. Using the link (4.3) we can rewrite:

$$
I_{i j k}\left[\boldsymbol{y}_{A}, r_{A}^{\prime}, r_{0}\right]=\lim _{\varepsilon \rightarrow 0}\left\{I_{i j k}^{\mathrm{DR}}\left[\boldsymbol{y}_{A}, r_{0}, \varepsilon, \ell_{0}\right]+\delta_{\boldsymbol{\eta}\left[\boldsymbol{y}_{A}, r_{A}^{\prime}, \varepsilon, \ell_{0}\right]} I_{i j k}\right\}
$$

where the modification $\delta_{\boldsymbol{\eta}} I_{i j k}=3 \sum_{A=1,2} m_{A} y_{A}^{\langle i} y_{A}^{j} \eta_{A}^{k\rangle}$ due to the latter shifts follows from the variation of the Newtonian mass octupole moment $I_{i j k}^{\mathrm{N}}=\sum_{A=1,2} m_{A} y_{A}^{\langle i} y_{A}^{j} y_{A}^{k\rangle}$ (valid in any dimension $d$ ) induced by $\boldsymbol{\eta}_{A}$. By construction, the poles $\sim 1 / \varepsilon$ cancel out between the two terms in the right-hand side of Eq. (4.5) so that the result is indeed finite (and does not depend on $\ell_{0}$ ) in the limit $\varepsilon \rightarrow 0$. The final scales it depends upon are the two UV scales $r_{A}^{\prime}$ and the IR scale $r_{0}$.

Now, the scales $r_{A}^{\prime}$ have been shown to be gauge constants, i.e. removable by a suitable gauge transformation, both in the equations of motion [37] and in the radiation field [3, 5]. We shall indeed check that these constants disappear when we compute the time derivatives of the octupole moment (4.5) by means of the 3PN equations of motion for insertion into the waveform. Our final invariant results, namely the gravitational modes $(3,3)$ and $(3,1)$ obtained in Sec. IVC, are thus independent of $r_{A}^{\prime}$.

On the other hand, the dependence on the IR constant $r_{0}$ offers the possibility of an interesting consistency check with the expression of the tails-of-tails for the mass octupole derived previously in Eq. (3.8b). Indeed, as we already mentioned, the tail-of-tail integrals depend on the constant $\tau_{0}=r_{0} / c$, where $r_{0}$ is defined by Eq. (2.7), in such a way that it will exactly cancel the constant $r_{0}$ coming from the expressions of the source multipole moments written in terms of the source parameters (i.e. the positions and velocities of the particles). That such a cancellation between tails-of-tails and source moments actually occurs has been proved very generally for any isolated matter system [2, 17]. This has also been explicitly checked in the case of point particle binaries for the mass quadrupole moment at 3PN order in Refs. [3, 5]. In Sec. [IVB we shall extend this check to the case of the mass octupole moment at $3 \mathrm{PN}$ order for general orbits in the center-of-mass frame.

\section{B. The 3PN mass octupole in the center-of-mass frame}

We have computed the 3PN mass octupole moment (4.5) of two point masses $\boldsymbol{y}_{A}$ for general orbits in an arbitrary frame. We then reduced that result to the frame of the center of mass defined by the nullity of the center-of-mass integral associated with the 3PN equations of motion, given by Eq. (2.13) in Ref. [38]. An interesting point about this calculation is that it requires the full $3 \mathrm{PN}$ relations between the variables in the center-of-mass frame and the relative variables, in contrast to what happens for the $3 \mathrm{PN}$ mass quadrupole moment where only the 2PN center-of-mass relations are needed [5].

The center-of-mass relations for point particle binaries take the form ${ }^{9}$

$$
\boldsymbol{y}_{1}=\left[X_{2}+\nu \Delta P\right] \boldsymbol{x}+\nu \Delta Q \boldsymbol{v}
$$

\footnotetext{
${ }^{9}$ Our notation for point particle binaries is as follows: $m_{A}$ stands for the two masses $(A=1,2) ; m=m_{1}+m_{2}$
} 


$$
\boldsymbol{y}_{2}=\left[-X_{1}+\nu \Delta P\right] \boldsymbol{x}+\nu \Delta Q \boldsymbol{v}
$$

where all the PN corrections are proportional to the symmetric mass ratio $\nu$ and the mass difference $\Delta=X_{1}-X_{2}$. The two dimensionless coefficients $P$ and $Q$ are given with the full 3PN precision in Eqs. (3.13)-(3.14) of Ref. [38].

As an interesting feature the gauge-constants $r_{A}^{\prime}$ appear at the $3 \mathrm{PN}$ order, in the coefficient $P$ only, in the form of the particular combination $r_{0}^{\prime \prime}$ defined by

$$
\Delta \ln r_{0}^{\prime \prime}=X_{1}^{2} \ln r_{1}^{\prime}-X_{2}^{2} \ln r_{2}^{\prime}
$$

(see Eq. (3.15) in Ref. [38]), due to the use of the full 3PN center-of-mass relations for this calculation. However, our final 3PN mass octupole source moment in the center-of-mass frame [Eqs. (4.9)-(4.11) below] will depend on $r_{A}^{\prime}$ through a combination that differs from that of Eq. (4.7), namely

$$
\ln r_{0}^{\prime}=X_{1} \ln r_{1}^{\prime}+X_{2} \ln r_{2}^{\prime} .
$$

Indeed we have found that the $r_{A}^{\prime}$ in the $3 \mathrm{PN}$ center-of-mass relations combine nicely with another combination of these constants in the 3PN mass octupole moment (4.5) for general orbits, i.e. after applying the required shift of the world lines (4.3) but before the center-of-mass reduction, so that the final center-of-mass expression of the mass octupole moment contains the classic combination of these constants as given by Eq. (4.8). This is perfectly consistent with the fact that the constants $r_{A}^{\prime}$ should in fine disappear from gauge-invariant results such as our final polarization $(3,3)$ and $(3,1)$ modes (4.18).

The mass octupole moment defined by Eq. (4.5) at the 3PN order, i.e. after the processes of dimensional regularization and renormalization by shifts of the world lines as reviewed in Sec. IVA, and reduced to the center-of-mass frame using Eqs. (4.6) with full 3PN precision, is finally of the form

$$
I_{i j k}=-\nu m \Delta\left\{A x_{\langle i} x_{j} x_{k\rangle}+B \frac{r}{c} v_{\langle i} x_{j} x_{k\rangle}+C \frac{r^{2}}{c^{2}} v_{\langle i} v_{j} x_{k\rangle}+D \frac{r^{3}}{c^{3}} v_{\langle i} v_{j} v_{k\rangle}\right\} .
$$

The coefficients for general orbits in the center-of-mass frame are found to be

$$
\begin{aligned}
A= & 1+\frac{1}{c^{2}}\left\{v^{2}\left(\frac{5}{6}-\frac{19}{6} \nu\right)+\frac{G m}{r}\left[-\frac{5}{6}+\frac{13}{6} \nu\right]\right\} \\
+ & \frac{1}{c^{4}}\left\{v^{4}\left(\frac{257}{440}-\frac{7319}{1320} \nu+\frac{5501}{440} \nu^{2}\right)+\frac{G m}{r}\left[v^{2}\left(\frac{3853}{1320}-\frac{14257}{1320} \nu-\frac{17371}{1320} \nu^{2}\right)\right.\right. \\
& \left.\left.+\dot{r}^{2}\left(-\frac{247}{1320}+\frac{531}{440} \nu-\frac{1347}{440} \nu^{2}\right)\right]+\frac{G^{2} m^{2}}{r^{2}}\left[-\frac{47}{33}-\frac{1591}{132} \nu+\frac{235}{66} \nu^{2}\right]\right\} \\
+ & \frac{1}{c^{5}}\left\{-\frac{56}{9} \frac{G^{2} m^{2} \nu \dot{r}}{r^{2}}\right\}
\end{aligned}
$$

for the total mass; $X_{A}=m_{A} / m$ for the two mass fractions; $\nu=X_{1} X_{2}$ for the symmetric mass ratio; $\mu=m \nu$ for the reduced mass; $\Delta=X_{1}-X_{2}$ for the relative mass difference; $\boldsymbol{x}=\left(x^{i}\right)=\boldsymbol{y}_{1}-\boldsymbol{y}_{2}$ and $\boldsymbol{v}=\left(v^{i}\right)=\mathrm{d} \boldsymbol{x} / \mathrm{d} t=\boldsymbol{v}_{1}-\boldsymbol{v}_{2}$ for the relative separation and velocity; $v^{2}=\boldsymbol{v}^{2}$ and $\dot{r}=\boldsymbol{n} \cdot \boldsymbol{v}$, where $\boldsymbol{n}=\boldsymbol{x} / r$ and $r=|\boldsymbol{x}|$. 


$$
\begin{aligned}
& +\frac{1}{c^{6}}\left\{v^{6}\left(\frac{3235}{6864}-\frac{7667}{1040} \nu+\frac{10319}{286} \nu^{2}-\frac{129707}{2288} \nu^{3}\right)\right. \\
& +\frac{G m}{r}\left[v^{4}\left(\frac{11633}{2640}-\frac{93167}{2640} \nu+\frac{5289}{110} \nu^{2}+\frac{203299}{2640} \nu^{3}\right)\right. \\
& +v^{2} \dot{r}^{2}\left(-\frac{841}{11440}+\frac{2039}{3432} \nu-\frac{121393}{11440} \nu^{2}+\frac{589981}{17160} \nu^{3}\right) \\
& \left.+\dot{r}^{4}\left(\frac{1}{208}-\frac{1379}{2288} \nu+\frac{44841}{11440} \nu^{2}-\frac{63699}{11440} \nu^{3}\right)\right] \\
& +\frac{G^{2} m^{2}}{r^{2}}\left[v^{2}\left(\frac{40497}{20020}-\frac{397027}{8580} \nu+\frac{120069}{2860} \nu^{2}-\frac{29429}{858} \nu^{3}\right)\right. \\
& \left.+\dot{r}^{2}\left(-\frac{60413}{120120}+\frac{20801}{1560} \nu-\frac{623351}{17160} \nu^{2}-\frac{70735}{3432} \nu^{3}\right)\right] \\
& \left.+\frac{G^{3} m^{3}}{r^{3}}\left[\frac{4553429}{229320}+\frac{902873}{15015} \nu-\frac{31673}{1716} \nu^{2}+\frac{27085}{5148} \nu^{3}-\frac{26}{7} \ln \left(\frac{r}{r_{0}}\right)-22 \nu \ln \left(\frac{r}{r_{0}^{\prime}}\right)\right]\right\} \\
& +\mathcal{O}\left(\frac{1}{c^{7}}\right) \\
& B=\frac{\dot{r}}{c}\{-1+2 \nu\} \\
& +\frac{\dot{r}}{c^{3}}\left\{v^{2}\left(-\frac{13}{22}+\frac{107}{22} \nu-\frac{102}{11} \nu^{2}\right)+\frac{G m}{r}\left[-\frac{2461}{660}+\frac{8689}{660} \nu+\frac{1389}{220} \nu^{2}\right]\right\} \\
& +\frac{1}{c^{4}}\left\{-\frac{12 G m \nu v^{2}}{5 r}+\frac{232 G^{2} m^{2} \nu}{15 r^{2}}\right\} \\
& +\frac{\dot{r}}{c^{5}}\left\{v^{4}\left(-\frac{2461}{5720}+\frac{37321}{5720} \nu-\frac{34627}{1144} \nu^{2}+\frac{127447}{2860} \nu^{3}\right)\right. \\
& +\frac{G m}{r}\left[v^{2}\left(-\frac{80629}{17160}+\frac{47979}{1144} \nu-\frac{167122}{2145} \nu^{2}-\frac{267081}{5720} \nu^{3}\right)\right. \\
& \left.+\dot{r}^{2}\left(\frac{5}{572}+\frac{1851}{1144} \nu+\frac{3059}{1560} \nu^{2}-\frac{299171}{17160} \nu^{3}\right)\right] \\
& \left.+\frac{G^{2} m^{2}}{r^{2}}\left[\frac{229}{9240}+\frac{33459}{440} \nu-\frac{1283}{330} \nu^{2}+\frac{2287}{264} \nu^{3}\right]\right\} \\
& +\mathcal{O}\left(\frac{1}{c^{6}}\right) \\
& C=1-2 \nu \\
& +\frac{1}{c^{2}}\left\{v^{2}\left(\frac{61}{110}-\frac{519}{110} \nu+\frac{504}{55} \nu^{2}\right)+\dot{r}^{2}\left(-\frac{1}{11}+\frac{4}{11} \nu-\frac{3}{11} \nu^{2}\right)\right. \\
& \left.+\frac{G m}{r}\left[\frac{1949}{330}+\frac{62}{165} \nu-\frac{483}{55} \nu^{2}\right]\right\} \\
& +\frac{1}{c^{4}}\left\{v^{4}\left(\frac{465}{1144}-\frac{35777}{5720} \nu+\frac{3057}{104} \nu^{2}-\frac{25071}{572} \nu^{3}\right)\right. \\
& +v^{2} \dot{r}^{2}\left(-\frac{197}{1430}+\frac{1637}{1430} \nu-\frac{849}{286} \nu^{2}+\frac{3063}{1430} \nu^{3}\right)
\end{aligned}
$$




$$
\begin{aligned}
+ & \frac{G m}{r}\left[v^{2}\left(\frac{91379}{17160}-\frac{169537}{5720} \nu+\frac{83211}{5720} \nu^{2}+\frac{504721}{8580} \nu^{3}\right)\right. \\
& \left.+\dot{r}^{2}\left(-\frac{3037}{3432}-\frac{125}{104} \nu+\frac{62143}{17160} \nu^{2}+\frac{113089}{8580} \nu^{3}\right)\right] \\
& \left.+\frac{G^{2} m^{2}}{r^{2}}\left[-\frac{34515967}{1261260}-\frac{75373}{8580} \nu+\frac{428669}{8580} \nu^{2}-\frac{62665}{2574} \nu^{3}+\frac{52}{7} \ln \left(\frac{r}{r_{0}}\right)\right]\right\} \\
+ & \mathcal{O}\left(\frac{1}{c^{5}}\right), \\
D=\frac{\dot{r}}{c} & \left\{\frac{13}{55}-\frac{52}{55} \nu+\frac{39}{55} \nu^{2}\right\} \\
+ & \frac{\dot{r}}{c^{3}}\left\{v^{2}\left(\frac{333}{1430}-\frac{3181}{1430} \nu+\frac{1849}{286} \nu^{2}-\frac{7247}{1430} \nu^{3}\right)\right. \\
& +\dot{r}^{2}\left(\frac{112}{2145}-\frac{224}{715} \nu+\frac{224}{429} \nu^{2}-\frac{448}{2145} \nu^{3}\right) \\
& \left.+\frac{G m}{r}\left[\frac{26641}{8580}-\frac{8341}{2860} \nu-\frac{1655}{156} \nu^{2}+\frac{24367}{4290} \nu^{3}\right]\right\} \\
+ & \mathcal{O}\left(\frac{1}{c^{4}}\right) \cdot
\end{aligned}
$$

Let us also give the result of the reduction to quasi-circular orbits. Introducing the postNewtonian parameter $\gamma=G m /\left(r c^{2}\right)$, we have

$$
\begin{aligned}
A^{\text {circ }} & =1-\gamma \nu+\gamma^{2}\left(-\frac{139}{330}-\frac{11923}{660} \nu-\frac{29}{110} \nu^{2}\right) \\
& +\gamma^{3}\left(\frac{1229440}{63063}+\frac{610499}{20020} \nu+\frac{319823}{17160} \nu^{2}-\frac{101}{2340} \nu^{3}-\frac{26}{7} \ln \left(\frac{r}{r_{0}}\right)-22 \nu \ln \left(\frac{r}{r_{0}^{\prime}}\right)\right) \\
& +\mathcal{O}\left(\frac{1}{c^{8}}\right) \\
B^{\text {circ }} & =\frac{196}{15} \gamma^{2} \nu+\mathcal{O}\left(\frac{1}{c^{6}}\right) \\
C^{\text {circ }} & =1-2 \nu+\gamma\left(\frac{1066}{165}-\frac{1433}{330} \nu+\frac{21}{55} \nu^{2}\right) \\
& +\gamma^{2}\left(-\frac{1130201}{48510}-\frac{989}{33} \nu+\frac{20359}{330} \nu^{2}-\frac{37}{198} \nu^{3}+\frac{52}{7} \ln \left(\frac{r}{r_{0}}\right)\right)+\mathcal{O}\left(\frac{1}{c^{6}}\right) \\
D^{\text {circ }} & =\mathcal{O}\left(\frac{1}{c^{4}}\right)
\end{aligned}
$$

We observe that the coefficients $A^{\text {circ }}$ and $C^{\text {circ }}$ for quasi-circular orbits are purely conservative, while the other ones, $B^{\text {circ }}$ and $D^{\text {circ }}$, are purely dissipative, i.e. due to radiation reaction. The results (4.11) extend Eq. (5.15a) in Ref. [12] to 3PN order.

As already mentioned, the IR constant $r_{0}$ coming from the MPM regulator (2.7) in the $3 \mathrm{PN}$ octupole moment is exactly compensated by the same constant $r_{0}=c \tau_{0}$ coming from the kernel of the mass octupole tail-of-tail integral displayed in Eq. (3.8b). Indeed we can check from Eqs. (4.9)-(4.10) that the octupole moment for general orbits depends on this 
constant through the combination

$$
I_{i j k}=\cdots-\frac{26}{21} \frac{G^{2} m^{2}}{c^{6}} I_{i j k}^{(2)} \ln r_{0}+\mathcal{O}\left(\frac{1}{c^{7}}\right),
$$

where we have re-expressed the 3PN coefficient by means of the Newtonian octupole $I_{i j k}^{\mathrm{N}}=\sum_{A} m_{A} y_{A}^{\langle i j k\rangle}$. The ellipsis denote all the other terms in Eqs. (4.9)-(4.10) which are independent of $r_{0}$. On the other hand, Eq. (3.8b) immediately gives

$$
U_{i j k}^{\text {tail-tail }}=\cdots+\frac{26}{21} \frac{G^{2} M^{2}}{c^{6}} M_{i j k}^{(5)} \ln r_{0}
$$

which is nicely consistent with the source moment (4.12) and shows that the octupole tailof-tail indeed compensates the $r_{0}$ present in the source octupole, at leading order. Recall that the coefficient $\alpha_{3}=26 / 21$ is a particular case of the general formula (3.9).

\section{The gravitational-wave octupole modes $(3,3)$ and $(3,1)$}

With the 3PN mass octupole source moment in hand, and using all the non-linear multipole interactions computed in Sec. III, we obtain the complete 3PN mass octupole radiative moment $U_{i j k}$. Now, recall that for non-spinning compact binaries, there is a clean separation of the modes $(\ell, m)$ into those with $\ell+m$ even, which depend only on the mass-type moments $U_{L}$, and those with $\ell+m$ odd, which depend only on the current-type ones $V_{L} \cdot{ }^{10}$ From the radiative octupole $U_{i j k}$ we can thus compute the associated modes $(3,3)$ and $(3,1)$ in the usual spin-weighted spherical-harmonic decomposition of the waveform (3.2) for quasi-circular orbits. For $\ell+m$ even we have, adopting the conventions of Refs. [12, 13],

$$
h_{\ell m}=-\frac{2 G}{R c^{\ell+2} \ell !} \sqrt{\frac{(\ell+1)(\ell+2)}{\ell(\ell-1)}} \alpha_{\ell m}^{L} U_{L},
$$

where the STF tensorial factor $\alpha_{\ell m}^{L}$ connects the usual basis of spherical harmonics $Y^{\ell m}$ to the set of STF products of unit direction vectors $\hat{N}_{L}$ [see Eq. (C10)]. Like in Refs. [12, 13], we pose

$$
h_{\ell m}=\frac{2 G m \nu x}{R c^{2}} \sqrt{\frac{16 \pi}{5}} H_{\ell m} \mathrm{e}^{-\mathrm{i} m \psi}
$$

where the post-Newtonian parameter $x=\left(\frac{G m \omega}{c^{3}}\right)^{2 / 3}$ is defined from the orbital frequency of circular motion $\omega$, and where $\psi$ denotes a particular phase variable related to the actual orbital phase of the binary, namely $\varphi=\int \omega \mathrm{d} t$, by

$$
\psi=\varphi-\frac{2 G M \omega}{c^{3}} \ln \left(\frac{\omega}{\omega_{0}}\right),
$$

\footnotetext{
10 This fact is more generally true for "planar" binaries, whose motion takes place in a fixed orbital plane, which is the case of spinning binaries with spins aligned or anti-aligned with the orbital angular momentum; see Ref. 13] for a proof.
} 
the constant frequency $\omega_{0}$ being directly linked to the time scale $b$ entering the relation (3.1) between harmonic and radiative coordinates:

$$
\omega_{0}=\frac{\mathrm{e}^{\frac{11}{12}-\gamma_{\mathrm{E}}}}{4 b}
$$

with $\gamma_{\mathrm{E}}$ denoting the Euler constant. The 1.5PN logarithmic phase modulation in Eq. (4.16) originates physically from tails that propagate in the far zone [8, 9]. The mass $M$ therein is the ADM mass; it must include the relevant post-Newtonian corrections, up to $1 \mathrm{PN}$ order in the present case (see Eq. (5.23) in Ref. [12]).

Our final results for the gravitational-wave modes $(3,3)$ and $(3,1)$ at order $3.5 \mathrm{PN}$ in the waveform for quasi-circular orbits read

$$
\begin{aligned}
H_{33}= & -\frac{3}{4} \mathrm{i} \sqrt{\frac{15}{14}} \Delta\left[x^{1 / 2}+x^{3 / 2}(-4+2 \nu)+x^{2}\left(3 \pi+\mathrm{i}\left[-\frac{21}{5}+6 \ln (3 / 2)\right]\right)\right. \\
+ & x^{5 / 2}\left(\frac{123}{110}-\frac{1838 \nu}{165}+\frac{887 \nu^{2}}{330}\right)+x^{3}\left(-12 \pi+\frac{9 \pi \nu}{2}\right. \\
& \left.+\mathrm{i}\left[\frac{84}{5}-24 \ln (3 / 2)+\nu\left(-\frac{48103}{1215}+9 \ln (3 / 2)\right)\right]\right) \\
+ & x^{7 / 2}\left(\frac{19388147}{280280}+\frac{492}{35} \ln (3 / 2)-18 \ln ^{2}(3 / 2)-\frac{78}{7} \gamma_{\mathrm{E}}+\frac{3}{2} \pi^{2}+6 \mathrm{i} \pi\left[-\frac{41}{35}+3 \ln (3 / 2)\right]\right. \\
& \left.\left.+\frac{\nu}{8}\left[-\frac{7055}{429}+\frac{41}{8} \pi^{2}\right]-\frac{318841}{17160} \nu^{2}+\frac{8237}{2860} \nu^{3}-\frac{39}{7} \ln (16 x)\right)\right] \\
+ & \mathcal{O}\left(\frac{1}{c^{8}}\right), \\
H_{31}= & \frac{\mathrm{i} \Delta}{12} \sqrt{14}\left[x^{1 / 2}+x^{3 / 2}\left(-\frac{8}{3}-\frac{2 \nu}{3}\right)+x^{2}\left(\pi+\mathrm{i}\left[-\frac{7}{5}-2 \ln 2\right]\right)\right. \\
+ & x^{5 / 2}\left(\frac{607}{198}-\frac{136 \nu}{99}-\frac{247 \nu^{2}}{198}\right)+x^{3}\left(-\frac{8 \pi}{3}-\frac{7 \pi \nu}{6}\right. \\
& \left.+\mathrm{i}\left[\frac{56}{15}+\frac{16 \ln 2}{3}+\nu\left(-\frac{1}{15}+\frac{7 \ln 2}{3}\right)\right]\right) \\
+ & x^{7 / 2}\left(\frac{10753397}{1513512}-2 \ln 2\left[\frac{212}{105}+\ln 2\right]-\frac{26}{21} \gamma_{\mathrm{E}}+\frac{\pi^{2}}{6}-2 \mathrm{i} \pi\left[\frac{41}{105}+\ln 2\right]\right. \\
+ & \left.\left.\left.\mathcal{O}\left(\frac{1}{c^{8}}\right) \cdot \frac{1738843}{19305}+\frac{41}{8} \pi^{2}\right)+\frac{327059}{30888} \nu^{2}-\frac{17525}{15444} \nu^{3}-\frac{13}{21} \ln x\right)\right] \\
& \left.+\frac{1}{8}\right)
\end{aligned}
$$

This extends Eqs. (9.4d) and (9.4f) in Ref. [12] by one-half PN order. We have verified the complete agreement in the test-mass limit $\nu \rightarrow 0$ with the corresponding modes computed by black-hole perturbation techniques and reported in Eqs. (4.9) of Refs. [39]. Notice that the latter work uses a phase variable which differs from our definition $\psi$ given by Eq. (4.16); 
in particular it happens to be different for each mode $(\ell, m) .{ }^{11}$

The other modes $(3,2)$ and $(3,0)$ are known at order $3 \mathrm{PN}$ but cannot be computed at order 3.5PN for now; this computation will have to wait for the completion of the current quadrupole moment $J_{i j}$ (currently known at order 2.5PN [12]) up to order 3PN. The derivation of the $3 \mathrm{PN}$ current quadrupole presents new difficulties with respect to the $3 \mathrm{PN}$ mass quadrupole or octupole moments, and will be left for future work.

\section{TAIL-INDUCED RESUMMED WAVEFORM}

\section{A. Resummation of IR logarithms}

By implementing the MPM algorithm, it has been shown by induction that the $n$-th post-Minkowskian coefficient in harmonic coordinates in an expansion when $r \rightarrow+\infty$ with $t_{r}=$ const involves powers of $1 / r$ and powers of the logarithm $\ln r$ up to $n-1$, so that its general structure at future null infinity reads $[14]^{12}$

$$
h_{(n)}^{\alpha \beta}(\mathbf{x}, t)=\sum_{\ell=0}^{+\infty} \hat{n}_{L}\left\{\sum_{\substack{1 \leqslant k \leqslant N \\ 0 \leqslant p \leqslant n-1}} \frac{(\ln r / b)^{p}}{r^{k}} F_{L(n) k p}^{\alpha \beta}\left(t_{r}\right)+R_{L(n) N}^{\alpha \beta}\left(r, t_{r}\right)\right\} .
$$

Here $h_{(n)}$ stands for the $n$-th order post-Minkowskian piece of the gravitational field either in the canonical or the general MPM algorithms reviewed in Sec. III. The functions $F_{L(n) k p}$ are complicated functionals of the canonical moments $\left\{M_{L}, S_{L}\right\}$, or the source and gauge moments, $\left\{I_{L}, J_{L}\right\}$ and $\left\{W_{L}, X_{L}, Y_{L}, Z_{L}\right\}$ respectively, depending on the chosen algorithm. The angular part is expressed with the STF products $\hat{n}_{L}$ of unit vectors $n^{i}=x^{i} / r$. The remainder $R_{L(n) N}\left(r, t_{r}\right)$ is $\mathcal{O}\left(1 / r^{N-\epsilon}\right)$, with $0<\epsilon \ll 1$ taking into account the fact that the expansion involves powers of $\ln r$. Finally the logarithms in Eq. (5.1) are conveniently rescaled by means of an arbitrary constant $b$, being understood that the functions $F_{L(n) k p}$ are themselves dependent on this constant $b$. Restricting our attention to the leading coefficient of $1 / r$ at infinity we write

$$
\begin{aligned}
h_{(n)}^{\alpha \beta} & =\frac{1}{r} z_{(n)}^{\alpha \beta}\left(\mathbf{n}, \ln r, t_{r}\right)+\mathcal{O}\left(\frac{1}{r^{2-\epsilon}}\right), \\
\text { with } \quad z_{(n)}^{\alpha \beta} & =\sum_{\ell} \hat{n}_{L} \sum_{p=0}^{n-1}\left(\ln \frac{r}{b}\right)^{p} F_{L(n) p}^{\alpha \beta}\left(t_{r}\right) .
\end{aligned}
$$

We shall refer to the logarithms generated in the far zone expansion of the metric at infinity as the $I R$ logarithms. They have their root in the famous logarithmic deviation of the retarded cones $t_{r}=$ const in harmonic coordinates with respect to the true null cones

11 It is related to ours in the test-mass limit $\left(m_{1} \rightarrow 0\right)$ by

$$
\psi_{\ell m}^{\mathrm{FI}}=\psi+2 x^{3 / 2}\left(\gamma_{\mathrm{E}}+\frac{3}{2} \ln (4 x)-\frac{17}{12}\right)+\psi_{\ell m}^{(3 \mathrm{PN})}
$$

with $\psi_{\ell m}^{(3 \mathrm{PN})}$ being defined by Eq. (4.5) of Ref. 39].

12 Recall that $t_{r} \equiv t-r$. In this section we pose $G=c=1$. 
$u=$ const, where $u$ is a null coordinate satisfying $g^{\mu \nu} \partial_{\mu} u \partial_{\nu} u=0$. The IR logarithms can be removed by a coordinate transformation order by order in the MPM expansion. We have already seen in Eqs. (3.1) -(3.2) that one can construct radiative coordinates that are free of any such IR logarithms. Radiative coordinates are such that $T_{R}=u+\mathcal{O}(1 / R)$.

In this subsection we consider a particular class of IR logarithms generated by tails. Recall that in the MPM algorithm the IR logarithms are produced by source terms behaving like $1 / r^{2}$ when $r \rightarrow+\infty$ with $t_{r}=$ const (where we include in the coefficient the usual dependence on powers of $\ln r$ ). Although source terms behaving like $1 / r^{k}$ with $k \geqslant 3$ do generate $1 / r$ contributions after application of the retarded integral [i.e. $\mathrm{FP}_{B=0} \square_{\mathrm{ret}}^{-1} \widetilde{r}^{B}$ in the notation of Eq. (2.6)], those are in the form of source-free retarded waves which do not contain IR logarithms; this is proved by Lemma 7.2 in Ref. [14].

Using the leading order behaviour $1 / r$ of $h_{(n)}$, Eqs. (5.2), we see that IR logarithms can only come from that part of the gravitational source term $\Lambda$ in Eq. (2.1a) which is quadratic in $h$, say $\Lambda=N(h, h)+\mathcal{O}\left(h^{3}\right)$ where $N(h, h)$ is bilinear in $h$ as well as its space-time derivatives $\partial h$ and $\partial^{2} h$. Thus IR logarithms come only from solving the equation

$$
\square h_{(n)}^{\alpha \beta}=\sum_{m=1}^{n-1} N^{\alpha \beta}\left(h_{(m)}, h_{(n-m)}\right)+\mathcal{O}\left(\frac{1}{r^{3-\epsilon}}\right) .
$$

Writing the Einstein equations in harmonic coordinates in terms of the gothic metric and using the asymptotic formula $\partial_{\mu} h_{(n)}^{\alpha \beta}=-k_{\mu} \partial_{t} h_{(n)}^{\alpha \beta}+\mathcal{O}\left(1 / r^{2-\epsilon}\right)$, where $t_{r}=$ const and $k_{\mu}=$ $\left(-1, n^{i}\right)$ denotes a null Minkowskian vector, yields [21, 22]

$$
\square h_{(n)}^{\alpha \beta}=\frac{1}{r^{2}}\left[4 M \partial_{t}^{2} z_{(n-1)}^{\alpha \beta}+k^{\alpha} k^{\beta} \sigma_{(n)}\right]+\mathcal{O}\left(\frac{1}{r^{3-\epsilon}}\right) .
$$

The second term in that expression is due to the re-radiation of gravitational waves by the stress-energy tensor of gravitational waves themselves. This term is responsible for the non-linear memory effect [22, 26-32] (see Sec. IIIA). The energy density $\sigma_{(n)}$ is proportional to the total gravitational-wave flux emitted to order $n$ and reads explicitly

$$
\sigma_{(n)}=\frac{1}{2} \sum_{m=1}^{n-1}\left(\eta_{\mu \rho} \eta_{\nu \sigma}-\frac{1}{2} \eta_{\mu \nu} \eta_{\rho \sigma}\right) \partial_{t} z_{(m)}^{\mu \nu} \partial_{t} z_{(n-m)}^{\rho \sigma} .
$$

As proved in Ref. 21] (see Lemma 2.1 there) the IR logarithms coming from the second term in Eq. (5.4) can be removed, order by order in the MPM iteration, by means of the gauge transformation with gauge vector

$$
\lambda_{(n)}^{\alpha}=\square_{\text {ret }}^{-1}\left[\frac{k^{\alpha}}{2 r^{2}} \int_{-\infty}^{t_{r}} \mathrm{~d} \tau \sigma_{(n)}(\mathbf{n}, \ln r, \tau)\right] .
$$

As the retarded integral is convergent there is no need to include the $\mathrm{FP}_{B=0}$ operation.

In this Appendix we shall be interested in the IR logarithms generated by the tail term associated with backscatter onto the static space-time curvature generated by the total ADM mass $M$ of the system - the first term in the right-hand side of Eq. (5.4). The mass is introduced in the formalism as the constant monopole moment $I \equiv M$ in the "canonical" linearized metric (2.2). To prove that $M$ enters the source term at any MPM order $n$ in the way shown in (5.4), we invoke our assumption that the matter system is stationary before 
some instant $-\mathcal{T}$ in the past. Under this assumption the relaxed Einstein equation (2.1a) for the quadratic metric in the stationary epoch $t \leqslant-\mathcal{T}$ tells us that $\Delta h_{(2)}^{\alpha \beta}=\mathcal{O}\left(1 / r^{4}\right)$ hence $h_{(2)}^{\alpha \beta}=\mathcal{O}\left(1 / r^{2}\right)$. By immediate recurrence, we conclude that $h_{(n)}^{\alpha \beta}=\mathcal{O}\left(1 / r^{n}\right)$ when $t \leqslant-\mathcal{T}$. From this we infer that $k_{\mu} k_{\nu} z_{(n)}^{\mu \nu}=0$ at any time for $n \geqslant 2$, since it is constant, due to the asymptotic form of the harmonic-gauge condition, and vanishes at early time $t \leqslant-\mathcal{T}$, while we have $k_{\mu} k_{\nu} z_{(1)}^{\mu \nu}=-4 M$. Thus, for any $n \geqslant 2$, the first term of Eq. (5.4) comes only from the coupling between the static part of $h_{(1)}$ and $h_{(n-1)}$.

We shall solve recursively and look for IR logarithms in the equation

$$
\square \bar{h}_{(n)}^{\alpha \beta}=\frac{4 M}{r^{2}} \partial_{t}^{2} \bar{z}_{(n-1)}^{\alpha \beta}+\mathcal{O}\left(\frac{1}{r^{3-\epsilon}}\right) .
$$

We add an overbar to emphasize that we are considering an approximation of the harmoniccoordinate MPM algorithm in which we neglect all the IR logarithms generated by the second term of Eq. (5.4). The iteration of Eq. (5.7) is achieved most easily in the frequency space. The time Fourier transform of a function $F(t)$ will be denoted by $\tilde{F}(\Omega) \equiv \mathcal{F}(F)(\Omega)$ with the convention

$$
\tilde{F}(\Omega)=\int_{-\infty}^{+\infty} \mathrm{d} t F(t) \mathrm{e}^{\mathrm{i} \Omega t}, \quad F(t)=\int_{-\infty}^{+\infty} \frac{\mathrm{d} \Omega}{2 \pi} \tilde{F}(\Omega) \mathrm{e}^{-\mathrm{i} \Omega t} .
$$

The integration of the relevant $1 / r^{2}$ source terms will be achieved in the Fourier domain with the help of the following formula:

$$
\begin{aligned}
\square_{\mathrm{ret}}^{-1}\left[\frac{\hat{n}_{L}}{r^{2}}\left(\ln \frac{r}{b}\right)^{p} F\left(t_{r}\right)\right] & =\frac{\hat{n}_{L}}{2(p+1) r} \int_{-\infty}^{+\infty} \frac{\mathrm{d} \Omega}{2 \pi} \frac{\tilde{F}(\Omega) \mathrm{e}^{-\mathrm{i} \Omega t_{r}}}{(-\mathrm{i} \Omega)}\left[\gamma_{\ell}^{(p+1)}(0, \Omega b)-\left(\ln \frac{r}{b}\right)^{p+1}\right] \\
& +\mathcal{O}\left(\frac{1}{r^{2-\epsilon}}\right)
\end{aligned}
$$

in which the function of two variables $\gamma_{\ell}(B, x)$ is defined by (with $\Gamma$ the Eulerian function)

$$
\gamma_{\ell}(B, x)=\frac{\Gamma(\ell+1+B) \Gamma(1-B)}{(-2 \mathrm{i} x)^{B} \Gamma(\ell+1-B)} .
$$

In Eq. (5.9) this function is differentiated $(p+1)$ times with respect to the first variable $B$, and then evaluated at $B=0$ and for $x=\Omega b$, defining thus

$$
\gamma_{\ell}^{(p+1)}(0, \Omega b) \equiv\left(\frac{\partial^{p+1} \gamma_{\ell}}{\partial B^{p+1}}\right)(0, \Omega b) .
$$

Again notice that the retarded integral (5.9) is convergent so there is no need to invoke a finite part operation like in Eq. (2.6). For the reader's convenience we provide the proof of the elementary formula (5.9) in Appendix B.

We can now obtain thanks to the elementary formula $(5.9)^{13}$ the leading order waveform $\bar{z}_{(n)}$ starting from the preceding one $\bar{z}_{(n-1)}$, both having general structures similar to

${ }^{13}$ Here we consider only the retarded integral of the source term, corresponding to the part $\bar{u}_{(n)}^{\alpha \beta}$ of the MPM algorithm [see Eqs. (2.5)-(2.6)], since the part $\bar{v}_{(n)}^{\alpha \beta}$ does not contain IR logarithms. 
Eqs. (5.2). This gives some recursion relations for the functions $F_{L(n) p}$ parametrizing their general structures. These are given in the Fourier domain for any $n \geqslant 2$ by

$$
\begin{array}{lrl}
\tilde{F}_{L(n) p}(\Omega)=\frac{2 \mathrm{i} M \Omega}{p} \tilde{F}_{L(n-1) p-1}(\Omega) & \text { for } 1 \leqslant p \leqslant n-1, \\
\tilde{F}_{L(n) 0}(\Omega)=-\sum_{q=0}^{n-2} \frac{2 \mathrm{i} M \Omega}{q+1} \gamma_{\ell}^{(q+1)}(0, \Omega b) \tilde{F}_{L(n-1) q}(\Omega) & \text { for } p=0 .
\end{array}
$$

Such recursion formulas are easily iterated with the result that

$$
\begin{array}{lr}
\tilde{F}_{L(n) p}=\frac{(2 \mathrm{i} M \Omega)^{p}}{p !} \tilde{F}_{L(n-p) 0} & \text { for } 1 \leqslant p \leqslant n-1, \\
\tilde{F}_{L(n) 0}=-\sum_{q=0}^{n-2} \frac{(2 \mathrm{i} M \Omega)^{q+1}}{(q+1) !} \gamma_{\ell}^{(q+1)}(0, \Omega b) \tilde{F}_{L(n-q-1) 0} & \text { for } p=0 .
\end{array}
$$

These results yield immediately the complete resummed waveform in the Fourier domain as follows. For convenience we denote by $\tilde{z}_{L}$ the STF piece with multipolarity $\ell$ in the full waveform. From the first result (5.13a) we then determine

$$
\tilde{z}_{L}^{\alpha \beta}(\ln r, \Omega)=\sum_{n=1}^{+\infty} \tilde{z}_{L(n)}^{\alpha \beta}(\ln r, \Omega)=\mathrm{e}^{2 \mathrm{i} M \Omega \ln \left(\frac{r}{b}\right)} \tilde{F}_{L 0}^{\alpha \beta}(\Omega),
$$

in which $\tilde{F}_{L 0}(\Omega)$ refers to the logarithmic-free part of the full waveform and is defined by $\tilde{F}_{L 0}=\sum_{n=1}^{+\infty} \tilde{F}_{L(n) 0}$. Next, the second result (5.13b) gives the logarithmic-free part of the waveform in terms of the linearized approximation which is nicely factorized out as

$$
\tilde{F}_{L 0}(\Omega)=\frac{\tilde{F}_{L(1) 0}(\Omega)}{\gamma_{\ell}(2 \mathrm{i} M \Omega, \Omega b)},
$$

where the denominator is made of the function $\gamma_{\ell}(B, x)$ now evaluated at $B=2 \mathrm{i} M \Omega$ and $x=\Omega b$, and where we have used the fact that $\gamma_{\ell}(0, x)=1$. Note that $\tilde{z}_{L(1)}(\Omega)=\tilde{F}_{L(1) 0}(\Omega)$ is the waveform at the linear order $n=1$. Finally, combining these two findings with the expression of the function (5.10) we obtain the final expression for our resummed tailmodified waveform:

$$
\bar{z}_{L}\left(\ln r, t_{r}\right)=\int_{-\infty}^{+\infty} \frac{\mathrm{d} \Omega}{2 \pi} \frac{\Gamma(\ell+1-2 \mathrm{i} M \Omega)}{\Gamma(\ell+1+2 \mathrm{i} M \Omega) \Gamma(1-2 \mathrm{i} M \Omega)} \tilde{z}_{L(1)}(\Omega) \mathrm{e}^{-\mathrm{i} \Omega t_{r}+2 \mathrm{i} M \Omega \ln (2|\Omega| r)+M|\Omega| \pi} .
$$

We gladly notice that the scale $b$ has cancelled out from the final result (5.16), in agreement with the fact that the iteration of the equation (5.7) does not make any reference to an arbitrary scale such as $b$. However, the dependence on $b$ is restored in radiative coordinates through the redefinition (3.1) of the retarded time $T_{R}$ at future null infinity, and the phase factor in Eq. (5.16) becomes $\exp \left[-\mathrm{i} \Omega T_{R}+2 \mathrm{i} M \Omega \ln (2|\Omega| b)+M|\Omega| \pi\right]$. We observe that all powers of $\ln r$ have been absorbed into $T_{R}$, but there are still powers of $\ln (2|\Omega| b)$ left. This motivates the change of phase variable introduced in Eq. (4.16), the constant frequency $\omega_{0}$ defined in Eq. (4.17) being chosen for convenience to minimize the number of terms in the waveform. Recall also that, as indicated by an overbar, the resummed waveform (5.16) does 
not constitute the complete resummed waveform in harmonic coordinates because we have systematically neglected the second term in Eq. (5.4). However, it motivates the introduction of factorized resummed waveforms [40] which we shall shortly review and complete in the next subsection for the case of the mass octupole waveform.

In fact, the solution (5.16) can be obtained without MPM iteration and resummation by computing directly the solution of a "scattering" problem,

$$
\square \bar{h}-\frac{4 M}{r} \partial_{t}^{2} \bar{h}=\bar{S},
$$

where the scattering barrier is the usual potential $M / r$, and where $\bar{S}$ is some effective source. To recover the solution (5.16), it suffices to impose that $\bar{S}$ decreases like $\mathcal{O}\left(1 / r^{3-\varepsilon}\right.$ ) at infinity. The most general solution of (5.17) can be constructed formally by convoluting the source $\bar{S}$ with the retarded Green function $G_{\text {ret }}\left(x-x^{\prime}\right)$ of the differential operator $\square-(4 M / r) \partial_{t}^{2}$. In Ref. [41], $G_{\text {ret }}\left(x-x^{\prime}\right)$ is computed explicitly using standard techniques of scattering theory [42]. In the limit $r \rightarrow+\infty$ at $t_{r}=$ const, it is essentially proportional to the retarded solution of the modified Whittaker equation and to the normalization coefficient of the solution regular at the origin, for $\Omega r \rightarrow 0$.

\section{B. Application to the octupole resummed waveform}

We conclude the paper with an application of our above computation of the 3.5PN accurate modes $h_{33}$ and $h_{31}$ to the effective-one-body (EOB) approach [43, 44] to analytically blend PN approximants and numerical-relativity (NR) results. Factorized resummed waveforms were introduced in Ref. [40] and consist of a physically motivated product of the Newtonian waveform, a relativistic correction coming from an effective source built from the EOB Hamiltonian, the resummed tail effects linked to propagation on a Schwarzschild background (see Sec. $\mathrm{VA}$ ), a residual tail dephasing $\mathrm{i} \delta_{\ell m}$ (complex), and finally the $\ell$-th power of a residual relativistic amplitude correction (thus purely real) denoted $\rho_{\ell m}$. The factorized resummed waveforms achieve better agreement with NR results than the conventional Taylor expanded PN waveforms [39, 40, 45, 46]. For $\ell+m$ even (corresponding to even-parity $\epsilon=0$ ) we have

$$
H_{\ell m}=H_{\ell m}^{\mathrm{N}} S_{\text {eff }} T_{\ell m} \mathrm{e}^{\mathrm{i} \delta_{\ell m}}\left(\rho_{\ell m}\right)^{\ell}
$$

The Newtonian approximation to any even-parity mode reads

$$
H_{\ell m}^{\mathrm{N}}=\frac{(-)^{(\ell-m+2) / 2}}{2^{\ell+1}\left(\frac{\ell+m}{2}\right) !\left(\frac{\ell-m}{2}\right) !(2 \ell-1) ! !}\left(\frac{5(\ell+1)(\ell+2)(\ell+m) !(\ell-m) !}{\ell(\ell-1)(2 \ell+1)}\right)^{1 / 2} s_{\ell}(\nu)(\mathrm{i} m)^{\ell} x^{\ell / 2-1},
$$

where we denote $s_{\ell}(\nu) \equiv X_{2}^{\ell-1}+(-)^{\ell} X_{1}^{\ell-1}$, see e.g. Eq. (9.5) in Ref. [12]. The effective source $S_{\text {eff }}=\frac{H_{\text {eff }}}{\mu c^{2}}$ is given at order $3 \mathrm{PN}$ by

$$
\begin{aligned}
S_{\mathrm{eff}}=1 & -\frac{x}{2}\left\{1+\left(-\frac{3}{4}-\frac{1}{3} \nu\right) x+\left(-\frac{27}{8}+\frac{11}{4} \nu\right) x^{2}\right. \\
& \left.+\left(-\frac{675}{64}+\left[\frac{4417}{72}-\frac{205}{96} \pi^{2}\right] \nu-\frac{17}{6} \nu^{2}+\frac{\nu^{3}}{81}\right) x^{3}+\mathcal{O}\left(\frac{1}{c^{8}}\right)\right\} .
\end{aligned}
$$


For $m \geqslant 0$, the leading Schwarzschild tail factor reads

$$
T_{\ell m}=\frac{\Gamma\left(\ell+1-2 \mathrm{i} k_{m}\right)}{\Gamma(\ell+1)} \mathrm{e}^{k_{m}\left[\pi+2 \mathrm{i} \ln \left(2 m \omega_{0} b\right)\right]},
$$

in which we denote $k_{m} \equiv G M m \omega / c^{3}$, with $M$ being the ADM mass to be inserted here at 1PN order like in Eq. (4.16) (see Eq. (5.23) in Ref. [12]), and where $\omega_{0} b$ is a pure real number to be found from Eq. (4.17). ${ }^{14}$ Note that the EOB tail factor (5.21) is motivated by the factorized resummed waveform (5.16). Indeed, the dominant Fourier component of the mode $h_{\ell m}$ defined by Eq. (4.15) is obtained by setting $\Omega=m \omega$ in the stationary phase approximation.

As for the residual dephasings $\delta_{33}$ and $\delta_{31}$, we find that they do not receive any finite mass correction $\mathcal{O}(\nu)$ at order $3 \mathrm{PN}$, and are therefore given by the sum of the expressions (22) and (24) of Ref. [40] truncated at order 2.5PN, and the 3PN contributions in the test mass limit computed in Eqs. (5.8c) and (5.8e) of Ref. [39], i.e. up to order 3PN:

$$
\begin{aligned}
& \delta_{33}=\frac{13}{10} y^{3 / 2}-\frac{80897}{2430} \nu y^{5 / 2}+\frac{39}{7} \pi y^{3}+\mathcal{O}\left(\frac{1}{c^{7}}\right), \\
& \delta_{31}=\frac{13}{30} y^{3 / 2}-\frac{17}{10} \nu y^{5 / 2}+\frac{13}{21} \pi y^{3}+\mathcal{O}\left(\frac{1}{c^{7}}\right),
\end{aligned}
$$

with $y=\left(G M \omega / c^{3}\right)^{2 / 3}$, and $M$ is the ADM mass.

Finally the most important inputs we provide in this application are the finite mass corrections to order $3 \mathrm{PN}$ of the amplitude factors $\rho_{33}$ and $\rho_{31}$ that will form the main blocks in the factorized resummation of waveforms [39, 40, 47]. These are straightforwardly computed from Eqs. (4.18); for completeness we report here the full 3PN expressions, extending at 3PN order Eqs. (52) and (54) in Ref. [40]:

$$
\begin{aligned}
\rho_{33}=1+ & \left(-\frac{7}{6}+\frac{2}{3} \nu\right) x+\left(-\frac{6719}{3960}-\frac{1861}{990} \nu+\frac{149}{330} \nu^{2}\right) x^{2} \\
+ & \left(\frac{3203101567}{227026800}-\frac{26}{7} \gamma_{\mathrm{E}}-\frac{13}{7} \ln (36 x)+\left[-\frac{129509}{25740}+\frac{41}{192} \pi^{2}\right] \nu\right. \\
\rho_{31}=1+ & -\left(-\frac{274621}{154440} \nu^{2}+\frac{12011}{46332} \nu^{3}\right) x^{3}+\mathcal{O}\left(\frac{1}{c^{8}}\right), \\
+ & \left(\frac{11706720301}{6129723600}-\frac{26}{63} \gamma_{\mathrm{E}}-\frac{13}{63} \ln (4 x)+\left[-\frac{9688441}{2084940}+\frac{41}{192} \pi^{2}\right] \nu\right. \\
& \left.+\frac{174535}{75816} \nu^{2}-\frac{727247}{1250964} \nu^{3}\right) x^{3}+\mathcal{O}\left(\frac{1}{c^{8}}\right) .
\end{aligned}
$$

This completes our application to EOB resummed waveforms.

\footnotetext{
${ }^{14}$ Our definition takes into account the modification of the phase given by Eq. (4.16). The factor used in Ref. [40] is thus related to ours by $T_{\ell m}^{\mathrm{DIN}}=T_{\ell m} \mathrm{e}^{2 \mathrm{i} k_{m} \ln \left(\omega / \omega_{0}\right)}$.
} 


\section{Acknowledgments}

We thank the Indo-French collaboration (IFCPAR) under which a major part of this work has been carried out. B.R.I. is grateful to IHES, France, and G.F. and L.B. to RRI, India for their support during the final stages of the project. We also thank Cyril Denoux for checking the typesetting of the longest equations.

\section{Appendix A: Integration of elementary cubic source terms}

In order to compute the tail-of-tail contributions to the waveform in the far zone, we need to control the dominant asymptotic behaviour at future null infinity of the (finite part of the) retarded integrals of relevant cubic-source piece, i.e. $\Lambda_{(3)}^{\alpha \beta}$ in the notation of Eq. (2.6). This problem is essentially solved in Appendix A of Ref. [2], but we shall provide here some more details and additional material.

For generic elementary source terms with multipolarity $\ell$ and radial dependence $1 / r^{k}$ $(k \geqslant 1)$ that involve a non-local integral whose kernel is a Legendre function $Q_{m}(x)$, we consider: ${ }^{15}$

$$
\Psi_{m, \ell, k}^{L}=\underset{B=0}{\mathrm{FP}} \square_{\mathrm{ret}}^{-1}\left[\widetilde{r}^{B} \hat{n}_{L} r^{-k} \int_{1}^{+\infty} \mathrm{d} x Q_{m}(x) F(t-r x)\right] .
$$

Depending on the values of $m, \ell$ and $k$, the far-zone expansion of $\Psi_{m, \ell, k}^{L}$ when $r \rightarrow \infty$ (with $t_{r}=$ const) takes one of the following forms. For $k=1$ and $m=\ell$ we have

$$
\Psi_{\ell, \ell, 1}^{L}=-\frac{\hat{n}_{L}}{8 r} \int_{0}^{+\infty} \mathrm{d} \tau F^{(-1)}\left(t_{r}-\tau\right)\left[\ln ^{2}\left(\frac{\tau}{2 r}\right)+4 H_{\ell} \ln \left(\frac{\tau}{2 r}\right)+4 H_{\ell}^{2}\right]+\mathcal{O}\left(\frac{1}{r^{2-\epsilon}}\right)
$$

where $H_{\ell}=\sum_{j=1}^{\ell} \frac{1}{j}$ is the $\ell$-th harmonic number, and $F^{(-1)}$ denotes the anti-derivative of $F$ that vanishes at $-\infty$. For $2 \leqslant k \leqslant \ell+2$ and $k \geqslant \ell+3$ respectively, we have

$$
\begin{aligned}
\Psi_{m, \ell, k}^{L} & =-\alpha_{m, \ell, k} \frac{\hat{n}_{L}}{r} F^{(k-3)}\left(t_{r}\right)+\mathcal{O}\left(\frac{1}{r^{2-\epsilon}}\right), \\
\Psi_{m, \ell, k}^{L} & =-\frac{\hat{n}_{L}}{r} \int_{0}^{+\infty} \mathrm{d} \tau F^{(k-2)}\left(t_{r}-\tau\right)\left[\beta_{m, \ell, k} \ln \left(\frac{\tau}{2 r_{0}}\right)+\gamma_{m, \ell, k}\right]+\mathcal{O}\left(\frac{1}{r^{2-\epsilon}}\right) .
\end{aligned}
$$

The formula (A4) and the explicit expression of $\alpha_{m, \ell, k}$ for arbitrary $m, \ell, k$, are obtained in Ref. [2], as well as the explicit expressions of $\beta_{m, \ell, k}$ and $\gamma_{m, \ell, k}$ for specific values of $k$ and $\ell$.

15 The Legendre function of the second kind $Q_{m}(x)$ considered here has a branch cut from $-\infty$ to 1 , and is defined by (the first equality being known as Neumann's formula)

$$
Q_{m}(x)=\frac{1}{2} \int_{-1}^{1} \mathrm{~d} z \frac{P_{m}(z)}{x-z}=\frac{1}{2} P_{m}(x) \ln \left(\frac{x+1}{x-1}\right)-\sum_{j=1}^{m} \frac{1}{j} P_{m-j}(x) P_{j-1}(x),
$$

where $P_{m}(z)$ is the usual Legendre polynomial whose Rodrigues' representation reads

$$
P_{m}(z)=\frac{1}{2^{m} m !} \frac{\mathrm{d}^{m}}{\mathrm{~d} z^{m}}\left[\left(z^{2}-1\right)^{m}\right] .
$$


Let us first recall the derivation of the coefficient $\alpha_{m, \ell, k}$, which is given for general values of $k$ and $\ell$ such that $2 \leqslant k \leqslant \ell+2$ by

$$
\alpha_{m, \ell, k}=\int_{1}^{+\infty} \mathrm{d} x Q_{m}(x) \int_{x}^{+\infty} \mathrm{d} z Q_{\ell}(z) \frac{(z-x)^{k-3}}{(k-3) !} .
$$

It is convenient to introduce as intermediate notation the $(k-2)$-th anti-derivative of $Q_{\ell}(x)$ that vanishes at $x=+\infty$, and given for $k \geqslant 3$ by

$$
Q_{\ell}^{(-k+2)}(x)=-\int_{x}^{+\infty} \mathrm{d} z Q_{\ell}(z) \frac{(x-z)^{k-3}}{(k-3) !} .
$$

For $k=2$ we naturally pose $Q_{\ell}^{(0)}(x)=Q_{\ell}(x)$. Recalling that $Q_{\ell}(z)$ behaves like $z^{-\ell-1}$ when $z \rightarrow+\infty$ we see that the integral in the right side is convergent when $k \leqslant \ell+2$. Antiderivatives of Legendre functions can straightforwardly be expanded on the basis of Legendre functions themselves by means of the recurrence relation $(\mathrm{d} / \mathrm{d} z)\left[Q_{\ell+1}(x)-Q_{\ell-1}(x)\right]=(2 \ell+$ 1) $Q_{\ell}(x)$. This leads to

$$
\begin{aligned}
Q_{\ell}^{(-k+2)}(x) & =(-)^{k} \sum_{j=0}^{k-2} C_{\ell, j}^{k-2} Q_{\ell+2 j-k+2}(x), \\
\text { with } \quad C_{\ell, j}^{k-2} & =(-)^{j}\left(\begin{array}{c}
k-2 \\
j
\end{array}\right) \frac{(2 \ell+2 j-2 k+3) ! !}{(2 \ell+2 j+1) ! !}(2 \ell-2 k+4 j+5),
\end{aligned}
$$

where $\left(\begin{array}{c}k-2 \\ j\end{array}\right)$ is the usual binomial coefficient. Hence the coefficient $\alpha_{m, \ell, k}$ reads

$$
\alpha_{m, \ell, k}=(-)^{k} \int_{1}^{+\infty} \mathrm{d} x Q_{m}(x) Q_{\ell}^{(-k+2)}(x)=\sum_{j=0}^{k-2} C_{\ell, j}^{k-2} j_{m, \ell+2 j-k+2}
$$

where the remaining integral is explicitly given by (see e.g. [48] $)^{16}$

$$
j_{m, p}=\int_{1}^{+\infty} \mathrm{d} x Q_{m}(x) Q_{p}(x)= \begin{cases}\frac{H_{m}-H_{p}}{(m-p)(m+p+1)} & \text { for } m \neq p \\ \frac{1}{2 m+1}\left(\frac{\pi^{2}}{6}-H_{m, 2}\right) & \text { for } m=p .\end{cases}
$$

We next tackle the case of the two other coefficients $\beta_{m, \ell, k}$ and $\gamma_{m, \ell, k}$, defined for generic values of $k$ and $\ell$ such that $k \geqslant \ell+3$ as double integrals,

$$
\begin{aligned}
\beta_{m, \ell, k} & =\int_{1}^{+\infty} \mathrm{d} x Q_{m}(x) J_{\ell, k}(x), \\
\gamma_{m, \ell, k} & =\int_{1}^{+\infty} \mathrm{d} x Q_{m}(x)\left[\left(\ln 2+H_{k-3}\right) J_{\ell, k}(x)-K_{\ell, k}(x)\right],
\end{aligned}
$$

${ }^{16}$ Notice that $\psi(m+1)-\psi(p+1)=H_{m}-H_{p}$ and $\psi^{\prime}(m+1)=\frac{\pi^{2}}{6}-H_{m, 2}$, where $\psi(x)$ is the usual logarithmic derivative of the Euler gamma function $\Gamma(x)$ and $H_{m, 2}=\sum_{j=1}^{m} \frac{1}{j^{2}}$ is the $m$-th generalized harmonic number of order 2 . 
with the following definitions:

$$
\begin{aligned}
& J_{\ell, k}(x)=\frac{1}{2} \int_{-1}^{1} \mathrm{~d} z P_{\ell}(z) \frac{(z-x)^{k-3}}{(k-3) !}, \\
& K_{\ell, k}(x)=\frac{1}{2} \int_{-1}^{1} \mathrm{~d} z P_{\ell}(z) \frac{(z-x)^{k-3}}{(k-3) !} \ln (x-z) .
\end{aligned}
$$

Similarly to Eq. (A7) we introduce the $(k-2)$ th anti-derivative of the Legendre polynomial $P_{\ell}(x)$ that vanishes at $x=-1$ and is defined by

$$
P_{\ell}^{(-k+2)}(x)=\int_{-1}^{x} \mathrm{~d} z P_{\ell}(z) \frac{(x-z)^{k-3}}{(k-3) !}
$$

together with $P_{\ell}^{(0)}(x)=P_{\ell}(x)$. For the Legendre polynomial we have exactly the same expansion as in Eqs. (A8), namely

$$
P_{\ell}^{(-k+2)}(x)=(-)^{k} \sum_{j=0}^{k-2} C_{\ell, j}^{k-2} P_{\ell+2 j-k+2}(x) .
$$

We start by writing $J_{\ell, k}(x)$ and $K_{\ell, k}(x)$ in a way appropriate for future integration over $x$ with some kernel $Q_{m}(x)$. Since the following integral is already known [48],

$$
\int_{1}^{+\infty} \mathrm{d} x Q_{m}(x)(x-1)^{\nu}=2^{\nu} \frac{[\Gamma(\nu+1)]^{2} \Gamma(m-\nu)}{\Gamma(m+\nu+2)},
$$

our strategy will consist in expressing the integrals (A13) as a sum of monomials $(x-1)^{\nu}$, with $\nu \in \mathbb{N}$ or $\mathbb{C}$. This is achieved by performing $k-3$ integrations by parts on the expressions (A13). Concerning $J_{\ell, k}(x)$ this results in

$$
J_{\ell, k}(x)=\frac{(-)^{k+1}}{2} \sum_{p=\ell+1}^{k-2} \frac{P_{\ell}^{(-p)}(1)}{(k-p-2) !}(x-1)^{k-p-2},
$$

where the coefficients of each of the monomials are built from the values at 1 of the antiderivatives of the Legendre polynomial. Adapting some formulas for the anti-derivatives of the Legendre polynomial in Ref. [48], we get the values at $z=1$ as

$$
P_{\ell}^{(-p)}(1)= \begin{cases}0 & \text { for } 1 \leqslant p \leqslant \ell \\ \frac{(-)^{\ell} 2^{p}(p-1) !}{(p+\ell) !(p-\ell-1) !} & \text { for } \ell+1 \leqslant p .\end{cases}
$$

Note that the case for $1 \leqslant p \leqslant \ell$ is a simple consequence of Rodrigues' formula (A2); accordingly, we have written the sum in Eq. (A17) as starting with $p=\ell+1$. Finally, the integral (A12a) over $z$ can be computed with the help of formula (A16) with $\nu=k-p-2$.

The treatment of $K_{\ell, k}(x)$ is more involved. It is based on the fact that $\ln (x-z)=$ $\frac{\mathrm{d}}{\mathrm{d} B}\left[(x-z)^{B}\right]_{B=0}$ where $B$ is a convenient complex parameter. The calculation of $K_{\ell, k}(x)$ is thus similar to that of $J_{\ell, k}(x)$ with one major difference: After the $k-3$ integrations by parts, there remains a $x$-dependent factor $(z-x)^{B}$ in the source of the integral, which 
becomes $B(z-x)^{B-1}$ after a last integration by parts. The presence of the prefactor $B$ prevents the appearance of logarithms $\ln (x-z)$ when applying the derivative $\mathrm{d} / \mathrm{d} B$ and taking the limit $B \rightarrow 0$. We obtain at the end:

$$
\begin{aligned}
K_{\ell, k}(x) & =\frac{\mathrm{d}}{\mathrm{d} B}\left[\frac{(-)^{k+1}}{2} \sum_{p=\ell+1}^{k-2} \frac{P_{\ell}^{(-p)}(1)}{(k-3) !} \frac{\Gamma(B+k-2)}{\Gamma(B+k-p-1)}(x-1)^{B+k-p-2}\right]_{B=0} \\
& +\frac{(-)^{k}}{2} \int_{-1}^{1} \mathrm{~d} z \frac{P_{\ell}^{(-k+2)}(z)}{z-x} .
\end{aligned}
$$

The last term in Eq. (A19) is expanded by means of relation (A15), whose validity extends to the case $k \geqslant \ell+3$, provided that we pose $P_{-m-1}(z) \equiv-P_{m}(z)$ and $(-2 m-1) ! ! \equiv$ $(-)^{m} /(2 m-1)$ !! for any non-negative integer $m$. Using also Neumann's formula (A1), we obtain

$$
\frac{1}{2} \int_{-1}^{1} \mathrm{~d} z \frac{P_{\ell}^{(-k+2)}(z)}{z-x}=(-)^{k+1} \sum_{j=0}^{k-2} C_{\ell, j}^{k-2} Q_{\ell+2 j-k+2}(z),
$$

where we have defined $Q_{-m-1}(z) \equiv-Q_{m}(z)$ for $m \in \mathbb{N}$. Like for $J_{\ell, k}(x)$, the next step consists in integrating over $x$ each of the monomials $(x-1)^{B+k-p-2}$ by means of formula (A16), but this time with a complex parameter $\nu=B+k-p-2$. After integration we apply the derivative with respect to $B$ and take the limit $B \rightarrow 0$ which is straightforwardly performed and yields in particular many terms involving the logarithmic derivative $\psi$ of the Euler gamma function $\Gamma$ [which relates, for integer arguments, to harmonic numbers through the equality $\psi(k+1)-\psi(1)=H_{k}$ ]. Note that the integration over $x$ of the last term in (A19) produces the coefficient $\alpha_{m, \ell, k}$; compare Eq. (A20) with Eqs. (A8) and (A10). However, the coefficient $j_{m, p}$ defined in Eqs. (A11) must now be extended to the case where $p$ is negative by posing $j_{m,-|p|-1}=-j_{m,|p|}$.

Finally we are in a position to write down the expressions for $\beta_{m, \ell, k}$ and $\gamma_{m, \ell, k}$ [besides that given by (A10) for $\alpha_{m, \ell, k}$ ] that are effectively used in the present work:

$$
\begin{gathered}
\beta_{m, \ell, k}=(-)^{k+\ell+1} 2^{k-3} \sum_{j=0}^{k-\ell-3} \frac{(j+\ell) !}{j !(j+2 \ell+1) !} \frac{(m-k+\ell+2+j) !}{(k-\ell-3-j) !} \frac{[(k-\ell-3-j) !]^{2}}{(m+k-\ell-2-j) !} \\
\gamma_{m, \ell, k}=(-)^{k+\ell} 2^{k-3} \sum_{j=0}^{k-\ell-3} \frac{(j+\ell) !}{j !(j+2 \ell+1) !} \frac{(m-k+\ell+2+j) !}{(k-\ell-3-j) !} \frac{[(k-\ell-3-j) !]^{2}}{(m+k-\ell-2-j) !} \times \\
\quad \times\left(H_{k-\ell-3-j}-H_{m+\ell+2-k+j}-H_{m+k-\ell-2-j}\right)+\alpha_{m, \ell, k} .
\end{gathered}
$$

\section{Appendix B: Proof of an elementary integration formula}

We derive the integration formula (5.9) used in Sec. VA. We start with the following formula, valid through analytic continuation for any $B \in \mathbb{C}$,

$$
\square_{\text {ret }}^{-1}\left[\left(\frac{r}{b}\right)^{B} \frac{\hat{n}_{L}}{r^{2}} F\left(t_{r}\right)\right]=\frac{\hat{n}_{L}}{2 r} \int_{0}^{+\infty} \mathrm{d} \tau F\left(t_{r}-\tau\right) \frac{g_{\ell}(B)(\tau / 2 b)^{B}-(r / b)^{B}}{B}+\mathcal{O}\left(\frac{1}{r^{2-\epsilon}}\right),
$$


where the function $g_{\ell}(B)$ is

$$
g_{\ell}(B)=\frac{\Gamma(\ell+1+B) \Gamma(1-B)}{\Gamma(1+B) \Gamma(\ell+1-B)} .
$$

This formula (B1) is deduced from Eq. (A.2) of Ref. [30] by performing the change of variable $s=\tau+r$, expanding the STF derivative operator $\hat{\partial}_{L}$ (see (A.15) in [30]), and keeping the leading term of order $1 / r$. Here we are working in a neighbourhood of the value of interest $B=0$; in particular, we suppose $\Re(B)<\epsilon$. We point out that the elementary integral (B1) is convergent for $B$ close to zero. As usual we assume stationarity in the remote past, which implies in this case that $F(t)=0$ when $t<-\mathcal{T}$. Thus, despite the apparent presence of a pole $1 / B$, the explicit expression (B1) is finite and regular as $B \rightarrow 0$. After differentiating

$p$ times with respect to $B$, the left-hand side of (B1) acquires $p$ powers of the logarithm of $r / b$, while the right-hand side is straightforwardly evaluated at $B=0$ with the help of the Leibniz rule. We find

$$
\begin{aligned}
\square_{\text {ret }}^{-1}\left[\frac{\hat{n}_{L}}{r^{2}}\left(\ln \frac{r}{b}\right)^{p} F\left(t_{r}\right)\right] & =\frac{\hat{n}_{L}}{2(p+1) r} \int_{0}^{+\infty} \mathrm{d} \tau F\left(t_{r}-\tau\right)\left(\frac{\partial^{p+1}}{\partial B^{p+1}}\left[g_{\ell}(B)\left(\frac{\tau}{2 b}\right)^{B}-\left(\frac{r}{b}\right)^{B}\right]\right)_{B=0} \\
& +\mathcal{O}\left(\frac{1}{r^{2-\epsilon}}\right) .
\end{aligned}
$$

We see that each application of the retarded integral operator $\square_{\text {ret }}^{-1}$ on a source term increases the maximal power of the logarithms by one unit. Since there is no logarithm in the linearized part $h_{(1)}$, we infer by recurrence that the $n$th MPM coefficient $h_{(n)}$ contains logarithms with maximal power $n-1$ (the reasoning is in fact valid for any piece $\sim 1 / r^{k}$ in the waveform [14]). Finally the formula [48]

$$
\int_{0}^{+\infty} \mathrm{d} \tau \tau^{B} \mathrm{e}^{\mathrm{i} \Omega \tau}=\frac{\Gamma(B+1)}{(-\mathrm{i} \Omega)^{B+1}},
$$

achieves the integration of Eq. (B33) in the frequency space. This yields

$$
\begin{aligned}
& \square_{\text {ret }}^{-1}\left[\frac{\hat{n}_{L}}{r^{2}}\left(\ln \frac{r}{b}\right)^{p} F\left(t_{r}\right)\right]= \\
& \quad \frac{\hat{n}_{L}}{2(p+1) r} \int_{-\infty}^{+\infty} \frac{\mathrm{d} \Omega}{2 \pi} \frac{\tilde{F}(\Omega) \mathrm{e}^{-\mathrm{i} \Omega t_{r}}}{(-\mathrm{i} \Omega)}\left(\frac{\partial^{p+1}}{\partial B^{p+1}}\left[\frac{g_{\ell}(B) \Gamma(B+1)}{(-2 \mathrm{i} \Omega b)^{B}}-\left(\frac{r}{b}\right)^{B}\right]\right)_{B=0}+\mathcal{O}\left(\frac{1}{r^{2-\epsilon}}\right),
\end{aligned}
$$

which coincides with Eq. (5.9).

\section{Appendix C: Modal decomposition of the non-linear memory}

The expressions for the non-linear memory terms given in the text can be recovered, after appropriate integrations by part, from the general formula known for any $\ell$ [31, 32]:

$$
\begin{aligned}
U_{L}^{\text {mem }} & =\mathcal{U}_{L}^{\text {mem }}+(\text { instantaneous contributions }), \\
\text { with } \quad \mathcal{U}_{L}^{\text {mem }} & =\frac{2 c^{\ell-2}(2 \ell+1) ! !}{(\ell+1)(\ell+2)} \int_{-\infty}^{T_{R}} \mathrm{~d} t \int \mathrm{d} \Omega \frac{\mathrm{d} E}{\mathrm{~d} t \mathrm{~d} \Omega} \hat{N}_{L},
\end{aligned}
$$


where the instantaneous contributions come from the latter integrations by part. For convenience these instantaneous terms have been transferred to the instantaneous part of the radiative moments [see Eqs. (3.3)]. They have been included into our explicit expressions at $3.5 \mathrm{PN}$ order in Sec. IIIA 1. Recall in addition that we have $V_{L}^{\mathrm{mem}}=0$ for any current moment (at any $\mathrm{PN}$ order).

Here, $\frac{\mathrm{d} E}{\mathrm{~d} t \mathrm{~d} \Omega}=\frac{R^{2} c^{3}}{32 \pi G}\left(\partial_{t} g_{i j}^{\mathrm{TT}}\right)^{2}$ is the gravitational-wave energy flux per solid angle unit; the other notations are the same as in Sec. III. The right-hand side may be put in a more explicit form by substituting to $g_{i j}^{\mathrm{TT}}$ its asymptotic expansion (3.2) and integrating over the solid angle. The last operation is achieved by means of the useful identity:

$$
\int \frac{\mathrm{d} \Omega}{4 \pi} \hat{n}_{L_{1}} \hat{n}_{L_{2}} \hat{n}_{L}=\frac{\ell_{1} ! \ell_{2} ! \ell !}{\left(\frac{\ell_{1}+\ell_{2}-\ell}{2}\right) !\left(\frac{\ell_{1}+\ell-\ell_{2}}{2}\right) !\left(\frac{\ell_{2}+\ell-\ell_{1}}{2}\right) !} \mathrm{STF}_{k_{1} \ldots k_{\ell}} \frac{\delta_{k_{1}}^{\left\langle i_{1}\right.} \ldots \delta_{k_{s}}^{i_{s}} \delta_{\left\langle j_{1}\right.}^{i_{s+1}} \ldots \delta_{j_{\ell_{1}-s}}^{\left.i_{\ell_{1}}\right\rangle} \delta_{j_{\ell_{1}-s+1}}^{k_{s+1}} \ldots \delta_{\left.j_{\ell_{2}}\right\rangle}^{k_{\ell}}}{\left(\ell+\ell_{1}+\ell_{2}\right) ! !}
$$

with $s=\left(\ell_{1}+\ell-\ell_{2}\right) / 2$ and $\left|\ell_{1}-\ell_{2}\right| \leqslant \ell \leqslant \ell_{1}+\ell_{2}$ (hence $s \leqslant \ell$ and $\left.s \leqslant \ell_{1}\right)$.

After some combinatorics we find

$$
\begin{aligned}
& \mathcal{U}_{L}^{\mathrm{mem}}= \frac{2 G}{c^{3}(\ell+1)(\ell+2)} \sum_{p, k} \frac{1}{c^{2 p} p ! k} \frac{(2 \ell+1) ! !}{(2 p+2 \ell+1) ! !}\left(\begin{array}{c}
\ell \\
k-p
\end{array}\right) \\
& \times \int_{-\infty}^{T_{R}} \mathrm{~d} \tau\left[d_{p k}^{\ell} U_{P\langle K-P}^{(1)} U_{L-[K-P]\rangle P}^{(1)}+\frac{e_{p k}^{\ell}}{c^{2}} V_{P\langle K-P}^{(1)} V_{L-[K-P]\rangle P}^{(1)}\right. \\
&\left.\quad+\frac{f_{p k}^{\ell}}{c^{2}} \varepsilon_{a b\left\langle i_{1}\right.} U_{\underline{a P} K-P-1}^{(1)} V_{L-[K-P]\rangle b P}^{(1)}\right] .
\end{aligned}
$$

Note that this expression is implicit because the radiative moments $U_{L}$ in the right-hand side contain themselves a memory contribution. The coefficient $d_{p k}^{\ell}$ reads

$$
d_{p k}^{\ell}=k-4 p \frac{2 p+2 \ell+1}{2 p+\ell-k}+\frac{2 p(p-1)(2 p+2 \ell+1)(2 p+2 \ell-1)}{(k-1)(2 p+\ell-k)(2 p+\ell-k-1)},
$$

if $\max (0,[(5-\ell) / 2]) \leqslant p$ and $\max (p, 2) \leqslant k \leqslant \min (p+\ell, 2 p+\ell-2)$ (with $[\cdots]$ denoting the integer part), and $d_{p k}^{\ell}=0$ otherwise. The other coefficients are given by

$$
e_{p k}^{\ell}=\frac{4 k(2 p+\ell-k)}{(k+1)(2 p+\ell-k+1)} d_{p k}^{\ell}
$$

and

$$
f_{p k}^{\ell}=\frac{8(k-p)}{(2 p+\ell-k+2)}\left[\frac{p(2 p+2 \ell+1)}{(k-1)(2 p+\ell-k)}-1\right],
$$

if $\max (0,[(4-\ell) / 2]) \leqslant p$ and $\max (p+1,2) \leqslant k \leqslant \min (p+\ell, 2 p+\ell-1)$, and $f_{p k}^{\ell}=0$ otherwise.

For completeness, we shall now provide the corresponding formula for the modal decomposition of the non-linear memory term. The complex waveform $h \equiv h_{+}-\mathrm{i} h_{\times}$can be decomposed onto an orthonormal basis of functions with spin-weight -2 defined over the unit sphere. Here we shall choose the set of spin-weighted spherical harmonics $Y_{-2}^{\ell m}(\Theta, \Phi)$ and use the same conventions as in Refs. [12, 13]:

$$
h=\sum_{\ell=2}^{+\infty} \sum_{m=-\ell}^{\ell} h_{\ell m} Y_{-2}^{\ell m}(\Theta, \Phi)
$$


The coefficients $h_{\ell m}$ may be written in terms of mass and current type radiative components $U_{\ell m}$ and $V_{\ell m}$ (corresponding to a decomposition in even and odd parity modes in the case on non-spinning compact binaries):

$$
h_{\ell m}=-\frac{G}{\sqrt{2} R c^{\ell+2}}\left[U_{\ell m}-\frac{i}{c} V_{\ell m}\right] .
$$

Those are related to the STF radiative moments by [15]:

$$
\begin{aligned}
& U_{\ell m}=\frac{4}{\ell !} \sqrt{\frac{(\ell+1)(\ell+2)}{2 \ell(\ell-1)}} \alpha_{\ell m}^{L} U_{L}, \\
& V_{\ell m}=-\frac{8}{\ell !} \sqrt{\frac{\ell(\ell+2)}{2(\ell+1)(\ell-1)}} \alpha_{\ell m}^{L} V_{L},
\end{aligned}
$$

where $\alpha_{L}^{\ell m}$ is the unique (constant) STF tensor such that

$$
\hat{N}_{L}=\sum_{m=-\ell}^{\ell} \alpha_{\ell m}^{L} Y^{\ell m}(\Theta, \Phi) .
$$

To compute the memory modes $\mathcal{U}_{\ell m}^{\text {mem }} \propto \alpha_{\ell m}^{L} \mathcal{U}_{L}^{\text {mem }}$, we insert the angular integral of Eq. (C1b) into formula (C9a) and contract $\alpha_{\ell m}^{L}$ with $\hat{N}_{L}$ using the property (C10) [31]. It remains to integrate a product of three harmonic functions. After writing them as Wigner matrices, we obtain an explicit expression for the required integral by means of standard integration formulas:

$$
\begin{aligned}
& \int \frac{\mathrm{d} \Omega}{4 \pi} \bar{Y}^{\ell m}(\Theta, \Phi) Y_{-2}^{\ell^{\prime} m^{\prime}}(\Theta, \Phi) \bar{Y}_{-2}^{\ell^{\prime \prime} m^{\prime \prime}}(\Theta, \Phi) \\
& \quad=(-)^{m+m^{\prime}}\left(\frac{(2 \ell+1)\left(2 \ell^{\prime}+1\right)\left(2 \ell^{\prime \prime}+1\right)}{(4 \pi)^{3}}\right)^{1 / 2}\left(\begin{array}{ccc}
\ell & \ell^{\prime} & \ell^{\prime \prime} \\
0 & 2 & -2
\end{array}\right)\left(\begin{array}{ccc}
\ell & \ell^{\prime} & \ell^{\prime \prime} \\
-m & m^{\prime} & -m^{\prime \prime}
\end{array}\right) .
\end{aligned}
$$

This yields for the memory mode $(\ell, m)$

$$
\begin{aligned}
& \mathcal{U}_{\ell m}^{\text {mem }}=\frac{G}{c^{3}}\left[\frac{(2 \ell+1)(\ell-2) !}{8 \pi(\ell+2) !}\right]^{1 / 2} \sum_{k \geqslant \max (0,4-\ell)} \sum_{\ell^{\prime}=\max \left(\left[\frac{k+1}{2}\right], 2\right)}^{\min \left(\left[\frac{k}{2}\right]+\ell, k+\ell-2\right)} \sum_{m^{\prime}=\max \left(-\ell^{\prime}, m+\ell^{\prime}-\ell-k\right)}^{\min \left(\ell^{\prime}, m+\ell-\ell^{\prime}+k\right)} \frac{(-)^{m^{\prime}}}{c^{k}} \times \\
& \times\left[\left(2 \ell^{\prime}+1\right)\left(2 k+2 \ell-2 \ell^{\prime}+1\right)\right]^{1 / 2}\left(\begin{array}{ccc}
\ell & \ell^{\prime} & k+\ell-\ell^{\prime} \\
0 & 2 & -2
\end{array}\right)\left(\begin{array}{ccc}
\ell & \ell^{\prime} & k+\ell-\ell^{\prime} \\
-m & m^{\prime} & m-m^{\prime}
\end{array}\right) \times \\
& \times\left[\int_{-\infty}^{T_{R}} \mathrm{~d} \tau U_{\ell^{\prime} m^{\prime}}^{(1)} \bar{U}_{k+\ell-\ell^{\prime} m^{\prime}-m}^{(1)}+\frac{1}{c^{2}} \int_{-\infty}^{T_{R}} \mathrm{~d} \tau V_{\ell^{\prime} m^{\prime}}^{(1)} \bar{V}_{k+\ell-\ell^{\prime} m^{\prime}-m}^{(1)}\right. \\
& \left.+\frac{\mathrm{i}}{c} \int_{-\infty}^{T_{R}} \mathrm{~d} \tau\left(U_{\ell^{\prime} m^{\prime}}^{(1)} \bar{V}_{k+\ell-\ell^{\prime} m^{\prime}-m}^{(1)}-V_{\ell^{\prime} m^{\prime}}^{(1)} \bar{U}_{k+\ell-\ell^{\prime} m^{\prime}-m}^{(1)}\right)\right]
\end{aligned}
$$

where the even- $k$ (odd- $k$ ) coefficients cancel each other for memory integrals over products of multipole moments with different (identical) parities, as one can check explicitly.

[1] L. Blanchet, T. Damour, B. R. Iyer, C. M. Will, and A. G. Wiseman, Phys. Rev. Lett. 74, 3515 (1995), gr-qc/9501027. 
[2] L. Blanchet, Class. Quant. Grav. 15, 113 (1998), erratum Class. Quant. Grav. 22, 3381 (2005), gr-qc/9710038.

[3] L. Blanchet, B. R. Iyer, and B. Joguet, Phys. Rev. D 65, 064005 (2002), erratum Phys. Rev. D, 71:129903(E), 2005, gr-qc/0105098.

[4] L. Blanchet, G. Faye, B. R. Iyer, and B. Joguet, Phys. Rev. D 65, 061501(R) (2002), erratum Phys. Rev. D, 71:129902(E), 2005, gr-qc/0105099.

[5] L. Blanchet and B. R. Iyer, Phys. Rev. D 71, 024004 (2004), gr-qc/0409094.

[6] L. Blanchet, T. Damour, G. Esposito-Farèse, and B. R. Iyer, Phys. Rev. Lett. 93, 091101 (2004), gr-qc/0406012.

[7] L. Blanchet, T. Damour, G. Esposito-Farèse, and B. R. Iyer, Phys. Rev. D 71, 124004 (2005), gr-qc/0503044.

[8] L. Blanchet, B. R. Iyer, C. M. Will, and A. G. Wiseman, Class. Quant. Grav. 13, 575 (1996), gr-qc/9602024.

[9] K. Arun, L. Blanchet, B. R. Iyer, and M. S. Qusailah, Class. Quant. Grav. 21, 3771 (2004), erratum Class. Quant. Grav., 22:3115, 2005, gr-qc/0404185.

[10] L. Kidder, L. Blanchet, and B. R. Iyer, Class. Quant. Grav. 24, 5307 (2007), arXiv:0706.0726.

[11] L. Kidder, Phys. Rev. D 77, 044016 (2008), arXiv:0710.0614 [gr-qc].

[12] L. Blanchet, G. Faye, B. R. Iyer, and S. Sinha, Class. Quant. Grav. 25, 165003 (2008), arXiv:0802.1249 [gr-qc].

[13] G. Faye, S. Marsat, L. Blanchet, and B. R. Iyer, Class. Quant. Grav. 29, 175004 (2012), arXiv:1204.1043 [gr-qc].

[14] L. Blanchet and T. Damour, Phil. Trans. Roy. Soc. Lond. A 320, 379 (1986).

[15] K. Thorne, Rev. Mod. Phys. 52, 299 (1980).

[16] L. Blanchet, Phys. Rev. D 51, 2559 (1995), gr-qc/9501030.

[17] L. Blanchet, Class. Quant. Grav. 15, 1971 (1998), gr-qc/9801101.

[18] O. Poujade and L. Blanchet, Phys. Rev. D 65, 124020 (2002), gr-qc/0112057.

[19] L. Blanchet, G. Faye, and S. Nissanke, Phys. Rev. D 72, 044024 (2005).

[20] J. M. Martín-García, A. García-Parrado, A. Stecchina, B. Wardell, C. Pitrou, D. Brizuela, D. Yllanes, G. Faye, L. Stein, R. Portugal, et al., xAct: Efficient tensor computer algebra for Mathematica (GPL 2002-2012), http://www.xact.es/.

[21] L. Blanchet, Proc. Roy. Soc. Lond. A 409, 383 (1987).

[22] L. Blanchet and T. Damour, Phys. Rev. D 46, 4304 (1992).

[23] L. Blanchet, G. Faye, and B. F. Whiting, Phys. Rev. D 89, 064026 (2014), arXiv:1312.2975 [gr-qc].

[24] L. Blanchet, G. Faye, and B. F. Whiting, Phys. Rev. D 90, 044017 (2014), arXiv:1405.5151 [gr-qc].

[25] L. Blanchet and T. Damour, Phys. Rev. D 37, 1410 (1988).

[26] L. Blanchet, Ph.D. thesis, Université Paris VI, Paris (1990).

[27] D. Christodoulou, Phys. Rev. Lett. 67, 1486 (1991).

[28] A. Wiseman and C. Will, Phys. Rev. D 44, R2945 (1991).

[29] K. Thorne, Phys. Rev. D 45, 520 (1992).

[30] L. Blanchet, Class. Quant. Grav. 15, 89 (1998), gr-qc/9710037.

[31] M. Favata, Phys. Rev. D 80, 024002 (2009), arXiv:0812.0069 [gr-qc].

[32] M. Favata, Phys. Rev. D 84, 124013 (2011), arXiv:1108.3121 [gr-qc].

[33] L. Blanchet, T. Damour, and G. Esposito-Farèse, Phys. Rev. D 69, 124007 (2004), grqc/0311052. 
[34] L. Blanchet and G. Faye, J. Math. Phys. 41, 7675 (2000), gr-qc/0004008.

[35] C. G. Bollini and J. J. Giambiagi, Phys. Lett. B 40, 566 (1972).

[36] G. 't Hooft and M. Veltman, Nucl. Phys. B44, 139 (1972).

[37] L. Blanchet and G. Faye, Phys. Rev. D 63, 062005 (2001), gr-qc/0007051.

[38] L. Blanchet and B. R. Iyer, Class. Quant. Grav. 20, 755 (2003), gr-qc/0209089.

[39] R. Fujita and B. Iyer, Phys. Rev. D 82, 044051 (2010), arXiv:1005.2266 [gr-qc].

[40] T. Damour, B. Iyer, and A. Nagar, Phys. Rev. D 79, 064004 (2009), arXiv:0811.2069 [gr-qc].

[41] H. Asada and T. Futamase, Phys. Rev. D 56, 6062 (1997), gr-qc/9711009.

[42] A. Messiah, Mécanique Quantique (Dunod, Paris, 1959).

[43] A. Buonanno and T. Damour, Phys. Rev. D 59, 084006 (1999), gr-qc/9811091.

[44] T. Damour and A. Nagar, in Mass and motion in general relativity, edited by L. Blanchet, A. Spallicci, and B. Whiting (Springer, 2011), p. 211.

[45] T. Damour and A. Nagar, Phys.Rev. D79, 081503 (2009), 0902.0136.

[46] A. Buonanno, Y. Pan, H. Pfeiffer, M. Scheel, and L. a. Buchman, Phys. Rev. D 79, 124028 (2009), 0902.0790.

[47] R. Fujita, Prog. Theor. Phys. 128, 971 (2012), arXiv:1211:5535 [gr-qc].

[48] I. Gradshteyn and I. Ryzhik, Table of Integrals, Series and Products (Academic Press, 1980). 